\title{
Effects of Sweeping Jet Actuator Parameters on Flow Separation Control
}

\author{
Mehti Koklu ${ }^{1}$ \\ NASA Langley Research Center, Hampton, VA 23681
}

\begin{abstract}
A parametric experimental study was performed with sweeping jet actuators (fluidic oscillators) to determine their effectiveness in controlling flow separation on an adverse pressure gradient ramp. Actuator parameters that were investigated include blowing coefficients, operation mode, pitch and spreading angles, streamwise location, and size. Surface pressure measurements and surface oilflow visualization were used to characterize the effects of these parameters on the actuator performance. 2D Particle Image Velocimetry measurements of the flow field over the ramp and hot-wire measurements of the actuator's jet flow were also obtained for selective cases. In addition, the sweeping jet actuators were compared to other well-known flow control techniques such as micro-vortex generators, steady blowing, and steady vortex-generating jets. The results confirm that the sweeping jet actuators are more effective than steady blowing and steady vortexgenerating jets for this ramp configuration. The results also suggest that an actuator with a wider jet spreading (110 vs. 70 degrees) placed closer (2.3 vs. 7 boundary layer thickness upstream) to the flow separation location provides better performance. Different actuator sizes obtained by scaling down the actuator geometry produced different jet spreading. Scaling down the actuator (based on the throat dimensions) from $6.35 \times 3.18 \mathrm{~mm}$ to $3.81 \times 1.9 \mathrm{~mm}$ resulted in similar flow control performance; however, scaling down the actuator further to $1.9 \times 0.95 \mathrm{~mm}$ reduced the actuator efficiency by reducing the jet spreading considerably. The results of this study provide insight that can be used to design and select the optimal sweeping jet actuator configuration for flow control applications.
\end{abstract}

\section{Introduction}

One of the technical challenges of the NASA Fixed Wing Project is to reduce aircraft drag with minimal impact on cruise performance [1]. Reducing the drag not only improves aerodynamic efficiency, but also improves the fuel efficiency of an aircraft thereby reducing emissions and operating costs. One viable approach to reduce drag is to reduce the wetted area of aircraft components while maintaining aerodynamic performance. This can be accomplished on an aircraft wing through the use of high-lift systems. High-lift systems enable a more efficient wing in flight while adding more lift for takeoff and landing operations; however, they are vulnerable to flow separation at high angles of attack and large flap deflections. Developing methods to control the flow separation could help simplify the high-lift systems, which in turn, could help reduce drag and overall weight.

\footnotetext{
${ }^{1}$ Aerospace Engineer, Flow Physics and Control Branch, MS 170, Member AIAA.
} 
Flow separation control methods are usually designed to delay or completely eliminate separation by energizing the decelerated near-wall fluid by adding either momentum or vorticity to the boundary layer. Near-wall momentum addition in the form of blowing has been a preferred and straightforward separation control technique that has been studied extensively [2]. An alternate approach involves adding streamwise vorticity to increase the boundary-layer mixing and to enhance the convective transport of the freestream momentum toward the near wall. Vorticity addition can be either passive or active. Passive micro-vortex generators (MVGs) [3] have been successfully utilized in both low-speed [4, 5] and highspeed [6] applications to control the boundary layer. Lin [7] performed a detailed review of vortex generator studies that used MVGs to control boundary-layer separation. Active vortex-generating methods such as vortex-generating jets (VGJs) [8] provide the benefit of passive vortex generators without having the associated drag penalty. VGJs also provide an opportunity to adjust the flow control parameters to accommodate changing flow conditions.

Sweeping jet (SWJ) actuators, i.e., fluidic oscillators, have been the subject of much research in the active flow control field due to their unique design features such as nonmoving parts, a simple structure, limited maintenance requirements, dimensional scalability, and high-frequency and high-momentum bandwidth. These actuators emit spatially and temporally oscillating jets without having any electromechanical moving components. In their recent review, Gregory and Tomac [9] provided a brief history of the fluidic oscillators from their development as fluid amplifiers to their current usage as flow control actuators. Internal and external flow structures created by a similar SWJ actuator used in the present study were investigated both experimentally and numerically [10, 11]. Koklu and Melton [12] also studied the flow field produced by an SWJ actuator using hotwire anemometry and particle image velocimetry (PIV) measurements and compared the jet characteristics to that of a turbulent free jet. They reported that SWJ actuators produce wider jet-spreading and higher velocity fluctuations compared to the turbulent free jets and act as vortex-generating jets in failure modes [12]. SWJ actuators successfully controlled flow separation on an adverse pressure gradient (APG) ramp [13]. It was revealed that the SWJ actuators are more efficient than steady blowing using straight and angled jets (VGJs). A number of studies in the literature showed that SWJ actuators are reliable and efficient flowcontrol devices for improving aerodynamic performance. These studies include, but are not limited to, the application of SWJ actuators on a $30^{\circ}$ swept-wing high-lift model [14], a V-22 wing-nacelle combination [15], wind turbine blades [16], trucks [17], bluff bodies [18], and recently a full-scale Boeing 757 vertical tail model [19].

The objective of this study is to investigate the effects of actuator operation mode, placement, and size on the performance of the SWJ actuator in controlling flow separation. The effect of each parameter will be evaluated and compared to determine an efficient actuator configuration. Surface pressure measurements and surface flow visualization will be presented to show the effects of these parameters. For some selected cases, 2D PIV measurements of the flow field over the APG ramp and hot-wire measurements of the actuator's jet velocity will be provided and discussed. 


\section{Experimental Setup}

\section{A. Facility and Instrumentation}

The experiments in this study were conducted in the NASA Langley 15-Inch Low-Speed Tunnel in support of the NASA Fixed Wing Project. A description of the tunnel was reported in Refs. $[4,13]$. The tunnel is a closed-return, atmospheric facility used primarily for fundamental flow-physics research. The wind tunnel model consists of a long aluminum splitter plate with an APG ramp model placed between $X=148 \mathrm{~cm}$ and $X=190 \mathrm{~cm}$ (see Fig. 1), where $X=0$ is the leading edge of the splitter plate. Test section top and side walls were part of the wind tunnel walls and no measurements were taken on these walls. The height of the ramp model was $7.6 \mathrm{~cm}$. The model spanned the entire tunnel width $(38.1 \mathrm{~cm})$ and the height of top wall from the upper splitter plate was approximately $25 \mathrm{~cm}$. The boundary layer was tripped near the leading edge of the splitter plate and tunnel side walls (at $X=25 \mathrm{~cm}$ ). The grit strip was made from a $2.5 \mathrm{~cm}$ wide piece of a course/leveling grade of sand paper (no. 60 grit). The pressure distribution over the upper splitter plate was adjusted to be uniform between $X=50 \mathrm{~cm}$ and $X=127 \mathrm{~cm}$ using supports in the upper tunnel wall to slightly modify the ceiling geometry.

The ramp model was instrumented with surface-static pressure ports $(0.5 \mathrm{~mm}$ ID) located along the centerline. The pressure ports were connected to an electronically scanned pressure module that communicated with a computer via Ethernet. The pressure transducer in the acquisition module can record a maximum of $6.9 \mathrm{kPa}$ with an accuracy of $\pm 0.4 \mathrm{kPa}$. Surface pressure data presented in this study were obtained by ensemble averaging 6400 pressure signals from each pressure port to provide excellent accuracy and filtering.

A previously developed flow visualization technique was used in this study [13]. The surface oilflow visualization was obtained using a mixture of aviation oil, kerosene oil, and nanosized silica particles. For this particular application, the aviation oil was mixed with kerosene in a 1:4 ratio by volume and hydrophobic and hydrophilic silica particles were added to the mixture ( $2 \%$ by weight). Aviation oil contains fluorescent pigment that glows under the ultra-violet (UV) light and acts as a tracing media. Contrary to the $\mathrm{TiO}_{2}$ particles that are used as powdered pigments, the silica particles in this method serve as an antisagging agent to keep the aviation oil on the ramp surface when the tunnel is turned off. Therefore, the oil does not flow under the influence of gravity and hence eliminates in-situ photographing requirements. On the other hand, since the tracing media is oil, similar to the fluorescent oil-film technique, the oil mixture does not dry out quickly. In this technique, the mixture was applied to black contact paper mounted on the ramp model. As the tunnel speed was adjusted to the specified test conditions, the mixture moved under the effect of local shear stresses and revealed the surface flow topology. Fluorescent pigment in the aviation oil glowed under UV lighting and produced luminous flow visualization images.

Time-averaged velocity field measurements were made using a 2D PIV system for selected cases. The PIV images were recorded using a $2560 \times 1600$ pixel camera with a $55 \mathrm{~mm}$ macro lens. The field of view was approximately $31 \mathrm{~cm}$ wide by $19 \mathrm{~cm}$ tall along the tunnel centerline. Image resolution was approximately $83 \mathrm{pixel} / \mathrm{cm}$. The light sheet was produced by a Nd:YLF dual-cavity high-speed laser $(2 \times 30 \mathrm{~mJ} /$ pulse $)$ operated at $0.7 \mathrm{kHz}$ repetition 
rate. The flow was seeded with 1-micron particles produced by a commercial fog generator. For the data presented, 1000 image pairs were used to compute the time-averaged values. Interrogation window size was $16 \times 16$ pixels with $75 \%$ overlapping, leading to $0.48 \mathrm{~mm}$ vector pitch in both directions. The data were processed using commercially available PIV analysis software [20].

\section{B. Flow Control Methods and Baseline Parameters}

In order to provide a reference flow-control case, an array of MVGs was used as a passive flow control device. The centerline pressure $\left(C_{p}\right)$ distribution obtained using MVG flow control was used as a target $C_{p}$ distribution for the SWJ actuators. The MVGs were lowprofile, trapezoidal-shaped with $0.48 \mathrm{~cm}$ height and $1.9 \mathrm{~cm}$ length similar to that described in Ref. [4]. There were six counter-rotating MVGs installed at X $=147 \mathrm{~cm}$ and oriented at $\pm 23^{\circ}$ angles with respect to the direction of the freestream flow. MVG orientation was guided by the previous flow separation control studies of Lin [7] and Jenkins et al. [4]. Although MVGs are preferably located near suction peaks to generate stronger vortices, the MVGs in this study were located at this particular location to make an unbiased comparison with other cases. The spanwise locations of MVGs were determined based on the SWJ actuator geometry to make direct comparison. MVGs were symmetric about the SWJ actuator axis (Fig. 2); therefore, the spacing of the MVG pairs was the same as the SWJ actuator $(6.35 \mathrm{~cm})$. The leading edge of the MVGs was placed at the exit of the diverging nozzle that is $2.1 \mathrm{~cm}$ away from the SWJ axis. It is known that the wall-bounded flow over the ramp model generates two large corner vortices that dominate the tunnel flow due to the tunnel sidewalls [see Refs. 4 and 13, also later in oilflow visualizations]. In this study, no attempt was made to control these corner vortices. Instead, we focused on the flow at the center of the ramp model between two corner vortices. Therefore, the actuators (MVGs and SWJs) were placed so that they only affect the center ramp flow (Figs.1 and 2). The effect of tunnel sidewalls on the flow over the Stratford ramp was investigated in Ref. [21] and it was found that the separation bubble is smaller and more three dimensional in the case of tunnel with sidewalls. It was also concluded that the results can be extended to the ramp flow without tunnel sidewalls.

An array of SWJ actuators comprised of three-SWJ actuators (Fig. 2) was used as an active flow control device to control flow separation on the APG ramp. The actuator array was arranged in a lateral line pattern such that the center of the middle actuator was positioned at the tunnel centerline (Fig. 1). The spacing between two adjacent SWJ actuators was $6.35 \mathrm{~cm}$. The actuator array was fabricated from acrylic material with a computer numeric controlled milling machine. For the baseline configuration, the actuator array was installed at $X=147$ $\mathrm{cm}$ similar to MVGs. The actuator exits were flush with the splitter-plate surface. The jet axis of the SWJ actuators pointed downstream at an angle of $30^{\circ}$ to the freestream flow. The throat of the SWJ actuators was $6.35 \mathrm{~mm}$ wide by $3.18 \mathrm{~mm}$ deep, i.e., the aspect ratio (width/depth) of the actuators is 2 . Note that the actuator throat is not the same as the actuator exit. The actuator throat is located slightly upstream of the actuator exit creating a diverging nozzle between the actuator throat and exit. Each actuator in the array shared the same plenum. The flow rate to the actuator array was controlled by a pressure regulator and monitored by a mass flow meter. The actuator parameters such as blowing coefficient, 
operation mode, pitch and spreading angles, streamwise location, and size were changed one at a time from the baseline configuration to determine the effect of each parameter in controlling flow separation.

The momentum coefficient, $C_{\mu}$, is frequently used as a scaling parameter in the literature and defined as $C_{\mu}=2 \times V R^{2} \times \frac{A_{\text {jet }}}{A_{\text {ref }}}$, where $V R$ is the ratio of the area average jet velocity to the freestream velocity, $A_{j e t}$ is the total area of the actuator throats, and $A_{r e f}$ is the reference area. Since the actuators were designed to act over an area equal to half the tunnel width around the centerline, the reference area is the product of the tunnel half-width $(19 \mathrm{~cm})$ and the length of the ramp model $(40.6 \mathrm{~cm})$. Usually, the actuator exit area and the jet exit velocity are used in the $C_{\mu}$ calculation. However, since the actuator exits are flush with the local surface, the area of the actuator exit varies depending on the configuration (i.e., actuators with different spreading/pitch angles have different exit areas). In addition, depending on the jet spreading, the jet flow may not always fill the entire diverging nozzle reducing the effective jet exit area. Because of these uncertainties, the area of the actuator throat was used as $A_{j e t}$ and the average jet velocity at the throat was used as $U_{j e t}$ in the calculation of $C_{\mu}$. This definition of $C_{\mu}$ is true for incompressible flows and deemed valid for the present study because the velocity out of the SWJ actuator (measured and found to be 68 $\mathrm{m} / \mathrm{s}$ for the majority of the cases) was less than the incompressibility limit except for the smallest sized actuators for the same $C_{\mu}$. The compressibility effects result in a lower jet velocity and hence lower true $C_{\mu}$. Therefore, we also employed a power coefficient term, $C_{\pi}$, which does not include any assumptions about the jet velocity. The power coefficient is defined as [22]: $C_{\pi}=\frac{Q \times P_{a c t}}{\frac{1}{2} \times \rho_{\infty} \times A_{r e f} \times U_{\infty}^{3}}$, where $Q$ is the total volumetric flow rate to all actuators and $P_{a c t}$ is the static pressure inside the actuator plenum. This coefficient allows one to evaluate the efficiency of an actuation system in terms of required power. Although $C_{\pi}$ and $C_{\mu}$ are proportional for most cases, $C_{\pi}$ becomes important when the actuator geometry is modified. Therefore, we will report the $C_{\pi}$ results only for the cases where there is a variation in the actuator internal geometry, which results in a different power requirement.

The actuator operation mode is defined as the three different blowing types obtained using the same actuators $[12,13]$. In the first mode, the SWJ actuators were operated in normal mode where oscillating jets are emitted at the exit. The oscillating jet swept back and forth between the exit-side walls generating highly unsteady jets. In the second operation mode, one of the feedback loops was blocked and the SWJ actuators emitted steady angled jets at the exit. This type of blowing generates steady streamwise vortices, and is referred to as VGJs in the literature. In the third operation mode, the SWJ actuators emitted steady straight jets similar to tangential blowing. The steady straight jet was accomplished by a slight modification to the SWJ actuator geometry as described in Ref. [13]. Consequently, all three active flow control methods were obtained using the same actuators, which enabled a direct comparison of three different flow control methods. 
The effect of actuator placement was studied by varying the pitch angle $(a)$, spreading angle $(\theta)$, and the streamwise location $\left(X_{S W J}\right)$ of the SWJ actuators. The pitch angle, which is the angle between the jet axis and the local flow in the vertical plane, was varied from $30^{\circ}$ as the baseline to $16^{\circ}$ and $45^{\circ}$ using commercially available angle blocks. One surface of the angle block was attached to the actuator-top surface, and the other surface was attached to the splitter plate. Since the splitter plate was parallel to the freestream flow at $X=147 \mathrm{~cm}$, the accuracy of the pitch angle was assured using the angle blocks. Although the pitch angle could be well defined and its installation was straightforward, the spreading angle of an SWJ actuator is affected by the fluid interactions inside the actuator and; therefore, is not easy to control. The spreading angle is analogous to the skew angle of a VGJ actuator; however, the skew angle of a VGJ actuator is usually constant, but the skew angle of an SWJ actuator varies repetitively while the jet oscillates at the exit. In addition, the spreading angle of an SWJ actuator represents the time-averaged skew angle at each side of the actuator centerline. The easiest way of varying the spreading angle is by varying the opening angle of the actuator diverging nozzle so that the jet spreading is confined between the nozzle sidewalls. However, it was reported that when the diverging nozzle between the actuator throat and exit was shortened, the spreading angle was reduced greatly [23]. Note that the shorter nozzle results in smaller exit area, although the actuator internal geometry and the throat are the same. Usually, a shorter diverging nozzle is preferred in order to eliminate any further losses in the diverging nozzle. Therefore, we chose this arrangement rather than the former to show that designing an actuator with shorter nozzle may not always be the best option for SWJ actuator applications. The third parameter associated with the actuator placement is the streamwise location of the SWJ actuators, $X_{S W J}$. It has been known that active flow control methods work best when they are placed close to the flow separation location. In order to study the effect of the actuator location, we compared two cases where the actuator array was installed at $X=147 \mathrm{~cm}$ and $X=157 \mathrm{~cm}$. Note that since $X=157 \mathrm{~cm}$ location was on the ramp section, the pitch angle $(\boldsymbol{a})$ was different from that of the baseline case.

The effect of actuator size on the actuator performance was investigated by varying the scale of the SWJ actuators based on its throat dimensions (expansion in both lateral and vertical directions) without changing the aspect ratio or any other parameters. The baseline actuator had $6.35 \times 3.18 \mathrm{~mm}$ throat size $(1 \mathrm{X}$ scale). This size was scaled down to $3.81 \times 1.9 \mathrm{~mm}$ $(0.6 \mathrm{X}$ scale $)$, and $1.9 \times 0.95 \mathrm{~mm}(0.3 \mathrm{X}$ scale $)$, respectively. Reducing the actuator size required more pressure, i.e., more power, to keep the same momentum coefficient. Therefore, the power coefficient, $C_{\pi}$, was also used in comparisons to incorporate the power requirement of different sized actuators.

\section{Results and Discussion}

\section{A. Separated Flow and Reference Flow Control with MVGs}

All of the experiments in this study were performed at a freestream velocity, $U_{\infty}$, of $42.8 \pm 0.2 \mathrm{~m} / \mathrm{s}$. This corresponded to an approximate Mach number of 0.12 . The boundarylayer thickness $(\delta)$ and the momentum thickness at $X=145 \mathrm{~cm}$ were previously reported as $21.2 \mathrm{~mm}$ and $2.46 \mathrm{~mm}$, respectively. The shape factor at this location was calculated as 1.3, indicating a turbulent boundary layer entering the test section. The Reynolds number based 
on the momentum thickness was 6600 (approximately $2 \times 10^{5}$ based on the ramp height). Figure 3 shows a typical centerline $C_{p}$ distribution of the separated flow over the APG ramp without flow control. The no-flow-control (NFC) case was tested several times to check data repeatability. The maximum standard deviations were observed mostly at the upstream stations near the suction peaks and measured to be less than $0.2 \%$. The standard deviations were less than $0.06 \%$ for the downstream regions where flow separation was observed. The NFC case has a suction peak near $X=153 \mathrm{~cm}$ followed by a decrease in suction pressure due to the adverse pressure gradient. The $C_{p}$ distribution indicates flow separation near $\mathrm{X}=$ $162 \mathrm{~cm}$ where a sudden change (plateauing) in the pressure distribution occurs. Downstream of $\mathrm{X}=183 \mathrm{~cm}$, the centerline suction pressure continues to drop as the separated flow reattaches to the ramp surface. The effect of MVGs on the $C_{p}$ distribution is also shown in this figure. The MVG data represents a target $C_{p}$ distribution to be achieved by the SWJ actuators. Strong vortices generated by MVGs reduce the influence of the sidewall vortices and allow the centerline flow to remain attached as the suction pressure gradually decreases under the influence of APG. MVGs increase the upstream suction pressure slightly and provide substantial pressure recovery by eliminating flow separation.

Figure 4 shows contours of spanwise vorticity with in-plane velocity vectors on the centerline of the ramp for the NFC case. The spanwise vorticity was nondimensionalized by the length of the ramp model and freestream velocity. The freestream flow is from left to right as shown by the velocity vectors, and the gray area represents the APG ramp model. As indicated by the vorticity contours, the shear layer separates near $\mathrm{X}=162 \mathrm{~cm}$ as is consistent with the $C_{p}$ distribution. The reversed-flow line in this figure indicates flow reattachment near $\mathrm{X}=180 \mathrm{~cm}$. A thin reversed-flow region (approximately $0.2 \delta$ maximum thickness) is expected because the Stratford ramp is usually designed to generate an APG that causes minimum shear stress over the pressure recovery region. Discrete local reversed-flow regions are observed near flow separation $(X=162 \mathrm{~cm})$ due to the thin reversed-flow region. Since the field of view was relatively large covering the entire ramp section, the camera resolution was not sufficient to capture the details of this thin region near the wall. In addition, the surface glare and the surface curvature also limited the near-wall velocity measurements. The same holds true for the discrete reversed flow regions near flow reattachment. Low-magnitude vorticity is observed near the reattachment location where the shear stress is minimal. The magnitude of the vorticity level gradually increases as the centerline flow fully reattaches to the ramp surface.

Surface oilflow visualization of the NFC case is presented in Fig. 5. In all flow visualization figures, the flow direction is from top to bottom and the tunnel longitudinal stations are labeled on the side. The image shows two large corner vortices on the upper portion of the ramp as indicated by two spiral nodes. Consistent with the $C_{p}$ distribution and PIV measurements, oil mixture collected near $X=162 \mathrm{~cm}$ indicating a flow separation location. In this figure, there is another oil collection site near $X=170 \mathrm{~cm}$. On the ramp model, the gravitational and shear stress forces compete with each other due to the ramp inclination and the reversed flow. Therefore, this second oil accumulation site can be interpreted as the location where gravity and the shear stress balance each other. The center of the reattachment node, where the oil movement is minimal due to the low shear stress, is located approximately at $\mathrm{X}=181 \mathrm{~cm}$. This is slightly $(\sim 1 \mathrm{~cm})$ downstream that of the PIV 
measurement possibly because the PIV could not capture the thin separated flow near $\mathrm{X}=$ $180 \mathrm{~cm}$ due to the camera resolution. The separated flow is highly three dimensional because of the interaction between the reversed flow, the corner vortices, and the reattachment node.

Separated flow compares well with the previously reported results with similar configurations $[4,13]$. Although the separation location seems to be similar, oilflow visualization by Jenkins et al. [4] showed about $2-3 \mathrm{~cm}$ shorter separation bubble possibly due to the slight variation in the tunnel configurations.

Figure 6 shows the surface flow visualization of the MVG flow control case. The double lines on top of the figure illustrate the spanwise distribution of MVGs. Note that MVGs were placed at $X=147 \mathrm{~cm}$ in the streamwise direction. The curved arrow pair was added to the figure to visualize the generated streamwise vortices. Strong vortices generated by MVGs eliminated flow separation and allowed the flow in the center of the ramp to remain attached. The corner vortices could be seen in the domain because the six-MVG configuration acted only over an area equal to half the tunnel width around the tunnel centerline. However, compared to the NFC case, these corner vortices have larger footprint in the streamwise direction due to the attached flow in-between.

Usually, the counter-rotating vortices are classified based on the common flow in-between. They are referred to as "common-flow-down" when the common flow is directed toward the wall, and "common-flow-up" when it is directed away from the wall. Two counter-rotating vortices generated by a pair of diverging MVGs were in common-flow-down configuration that brought higher-momentum fluid from the outer boundary layer toward the wall. On the other hand, two counter-rotating vortices generated by a pair of converging MVGs were in a common-flow-up configuration that caused the local flow to lift off the ramp surface as indicated by the longitudinal flow detachment lines. The flow-detachment lines appear as dark lines when the circulation of the counter-rotating vortices are strong enough to lift the oil material off the surface or appear as stagnant oil lines when the circulation of the counter-rotating vortices are not strong enough to move the oil material.

On each side of the centerline, there are two small regions of recirculating flow formed by vortex pairs in the common-flow-up configuration along the flow-detachment lines near $\mathrm{X}=$ $162 \mathrm{~cm}$. Interestingly, their locations perfectly match with the flow separation location. Here, the local flow directed away from the wall thickens the boundary layer, which separates locally under the effect of APG, and generates these pockets of recirculating regions. A similar structure was also observed downstream of vortex pairs in the commonflow-up configuration generated by high profile VGs in experimental [5] and numerical [24] studies. The stagnant oil upstream and downstream of these pockets indicates the flowdetachment line. Spacing between two-detachment lines was $6.35 \mathrm{~cm}$, which was the spacing between two converging MVG pairs; however, these pockets were approximately 5 $\mathrm{cm}$ apart due to the corner vortices. 


\section{B. The Effect of $C_{\mu}$ and the Baseline Case}

The effect of the momentum coefficient $\left(C_{\mu}\right)$ was studied previously on a similar ramp model [13]. Since the target $C_{p}$ distribution (MVG pressure distribution) and the actuator configuration is different, the effect of the momentum coefficient was repeated here to find a baseline value for $C_{\mu}$ to be used with other parameters. Beginning from a low flow rate $\left(C_{\mu}\right.$ $=0.1 \%), C_{\mu}$ was gradually increased to achieve the target $C_{p}$ distribution of the MVG flow control case. Figure 7 shows that $C_{\mu}$ has a significant impact on the $C_{p}$ distribution. Higher $C_{\mu}$ values resulted in a higher upstream-suction peak and more downstream-pressure recovery. A $C_{p}$ distribution comparable to that of the MVG flow control case was obtained for $C_{\mu}=0.4 \%$. Therefore, $C_{\mu}=0.4 \%$ was inferred to be the minimum needed to recover the pressure losses due to flow separation on the APG ramp and was used as the baseline $C_{\mu}$ value in this study. Excessive blowing resulted in additional pressure recovery; however, the efficiency of the actuator system was greatly reduced.

Figure 8 exhibits the time-averaged contours of spanwise vorticity with in-plane velocity vectors for the baseline flow control case. The baseline case maintained attached flow over the APG ramp with the following parameters: $Q=4.15 \mathrm{~L} / \mathrm{s}, C_{\mu}=0.4 \%, \boldsymbol{a}=30^{\circ}, \theta=110^{\circ}$, and $X_{S W J}=147 \mathrm{~cm}$. We observed negative near-wall vorticity for the entire region, which is typical of attached flow. The contours of turbulent shear stress, $R x y$, for NFC and baseline cases are given in Fig. 9a and Fig. 9b, respectively. The $R x y$ term was normalized by the square of the freestream velocity, $U_{\infty}$. Separated flow over the hump model produced highly turbulent regions. Maximum shear stress appears to be slightly upstream of flow reattachment. Note how the application of SWJ actuators with their baseline configuration reduced the turbulent shear stress by eliminating flow separation. Figure 10 compares the time averaged velocity profiles at five streamwise locations for the NFC and baseline flow control cases. These velocity profiles were extracted from the PIV measurements at the stations shown by the dashed lines. The baseline case has much fuller velocity profile upstream of flow separation due to the energy addition by the SWJ actuators. The energized boundary layer resists the adverse pressure gradient further, while the boundary layer is about to separate for the NFC case near $X=162 \mathrm{~cm}$. As we travel downstream $(X=170$ $\mathrm{cm}$ ), the boundary layer becomes thicker for the baseline case and reverse flow is evident for the NFC case. Further downstream, the boundary layer continues to develop as the centerline flow is attached to the ramp surface; however, the baseline case still has fuller velocity profile.

Figure 11 presents the surface flow visualization for the baseline case. Compared to the previous flow visualization results (Figs. 5 and 6), the flow pattern was visualized immediate downstream of the $\mathrm{SWJ}$-actuator exits (from $\mathrm{X}=148 \mathrm{~cm}$ ) to observe how the generated vortices travel downstream before reaching the APG region. As the flow visualization in Fig. 11 shows, the SWJ actuators maintained attached flow in the center of the ramp. Two large corner vortices appear on the upper portion of the ramp. Their sizes were similar to that of the MVG case since no attempt was made to control them. While the jet oscillates at the SWJ actuator exit, the interaction between the oscillating jet and the freestream flow generates a spatially oscillating streamwise vortex around the actuator centerline. The spatially oscillating streamwise vortex is seen as a counter rotating vortex pair in the oilflow 
visualization images because they only show the time averaged effect. The counter-rotating vortex pairs generated by the same actuators are in common-flow-down configuration. These vortices are generated at two different periods of the jet oscillation cycle; therefore, they are out of phase and do not cancel each other's effect [13]. On the other hand, the counter rotating vortex pairs generated by adjacent actuators are in common-flow-up configuration. As a result, the generated vortex structure by the SWJ actuators is similar to the MVG case. In other words, the same number of vortices with the same rotation was generated at the same locations. Although the generated vortices were very similar to that of MVGs, we did not observe any small pockets of recirculating flow. The clear detachment lines in this figure indicate that the vortices generated by the SWJ actuators may be stronger than that of MVGs. Note that the clear flow detachment lines between two SWJ actuators denote only the time-averaged vortices generated by the SWJ actuators. The SWJ actuators instantaneously generate vortices in the entire region between the actuator-exit sidewalls due to the continuous jet oscillation. In contrast to the MVG control case, where steady streamwise vortices were generated, the spanwise-varying vortices generated by the SWJ actuators reduce any local boundary layer growth thereby eliminating any stagnant oilflow region.

\section{The Effect of Actuator Operation Modes}

The actuator operation mode refers to operating the same actuator in different ways to obtain three well-known active flow control methods: steady-straight jet (STJ), steady-angled jet (VGJ), and unsteady-oscillatory jet (SWJ). The SWJ actuators were used to produce three types of jets and all other parameters were set to their baseline values. Using the same actuator for both steady and unsteady jets enables a more direct comparison since it eliminates possible variations in actuator parameters such as location, orientation, orifice spacing, Ajet, and others, which may lead to biased conclusions. The actuator operation mode was studied previously on a similar ramp model [13]. However, since the target $C_{p}$ distribution (MVG pressure distribution) and the actuator configuration were different, the actuator operation mode was repeated to make a direct comparison with different actuator operation modes. The three operation modes only required a slight modification in the internal actuator geometry (such as blocking the feedback loops); therefore, the exit jet velocities were the same, but there were some variations in the plenum pressures. The plenum pressures for the same momentum coefficient $\left(C_{\mu}=0.4 \%\right)$ were $6.9 \mathrm{kPa}, 5.86 \mathrm{kPa}$, and $4.76 \mathrm{kPa}$ for the SWJ, VGJ, and STJ modes, respectively. As expected, blocking the feedback loops reduces the pressure losses inside the actuator. The $C_{p}$ distribution in Fig. 12a shows the comparison of these three flow control methods for a fixed momentum coefficient $\left(C_{\mu}=0.4 \%\right)$. This figure and the remaining $C_{p}$ distribution figures will focus on the separated flow region between $\mathrm{X}=162 \mathrm{~cm}$ and $\mathrm{X}=190 \mathrm{~cm}$. The $C_{p}$ distribution indicates an offset between the VGJ and SWJ actuators in the entire separated flow region. Although the VGJ mode did not have an optimal design compared to a specifically designed VGJ, the SWJ actuators performed better than the VGJ actuators for similar configurations. The superiority of the SWJ actuators over the VGJ actuators was attributed to the unsteady streamwise vortices generated by the SWJ actuators acting on both sides of the actuators, whereas the VGJ actuators generated steady vortices acting only on one side. These unsteady and spatially oscillating vortices resulted in increased coverage, more pressure 
recovery, and reduced flow separation [13]. As is consistent with data reported previously, the STJ actuators are the least effective method because they only energize the boundary layer by momentum addition. On the other hand, the VGJ and SWJ actuators generate streamwise vortices that provide more pressure recovery by distributing the high-momentum fluid in the boundary layer through mixing.

Different operation modes require different plenum pressures to keep the momentum coefficient the same. Since the actuator power consumption is directly related with the pressure, the effect of the operation mode was also evaluated at a fixed power coefficient, $C_{\pi}$. The tunnel parameters were constant for all cases; therefore, $C_{\pi}$ was kept constant by fixing the product of the plenum pressure and the actuator volume flow rate (i.e., $Q \times P_{a c t}$ ). For $C_{\pi}=0.8 \%$, the momentum coefficient is the same for the SWJ actuators but increases slightly for the VGJ and STJ actuators (Table 1). On the other hand, the pressure requirement to maintain the same $C_{\pi}=0.8 \%$ decreases (flow rate requirement increases) for the VGJ and STJ actuators (Table 1). Although the slight increase in $C_{\mu}$ resulted in better pressure recovery (Fig. 12b) when compared to the results for $C_{\mu}=0.4 \%$, the conclusion about the STJ actuators being the least effective and the SWJ actuators being the most effective is still valid.

The reason for the reduced performance of the VGJ and STJ modes can be explained by examining the surface oilflow visualization. Figure 13 shows the surface-flow visualization for the VGJ mode with all parameters set to their baseline values. The arrows at the top of this figure represent the location of the actuator exits as well as the blowing direction. The VGJ actuators created steady jets with a constant skew angle toward the left-hand side creating clockwise-rotating vortices (looking upstream). Since all three actuators blew in the same direction, the vortices were corotating as depicted by the curved arrows in the figure. Due to the left-hand-side blowing, we observe a bias toward that side and an accompanying smaller corner vortex. The flow visualization in Fig. 13 illustrates thicker oilflow patterns between two adjacent actuators beginning from $X=160 \mathrm{~cm}$ and extending to $X=175 \mathrm{~cm}$. The thicker oil flow indicates low-shear stress that is unable to move the oil material downstream. This region corresponds to the region where we observed a larger offset between the SWJ and VGJ actuators in the $C_{p}$ distribution (Fig. 12). In these regions, the VGJ actuators were unable to energize the boundary layer resulting in a thicker boundary layer (low shear stress) and causing pressure loss. Therefore, in order to recover the pressure losses between two adjacent actuators, more actuators are needed in the spanwise direction.

Surface oilflow visualization for the STJ actuators is presented in Fig. 14. The arrows represent the actuator locations, where the actuators blew jets parallel to the freestream flow in the horizontal plane. Note that the jet axis had a $30^{\circ}$ angle (pitch angle) in the vertical plane. Movement of the oil mixture due to the blown jet is displayed as darker regions near the actuator exits. A stagnant oilflow region is observed downstream of $X=166 \mathrm{~cm}$ extending to $X=182 \mathrm{~cm}$ indicating flow separation. Although the local flow is separated near $\mathrm{X}=162 \mathrm{~cm}$, we did not observe any stagnant oilflow possibly because the flow-driven oil "cleared out" the stagnant oil. Similar oil collection was also observed in the NFC case (Fig. 5), but the oil mixture did not move further downstream due to the balance between the gravity and the local shear stress. Beginning from $X=160 \mathrm{~cm}$, we observed thicker oilflow 
patterns (low shear stress) due to the thicker boundary layer similar to the flow visualization of the VGJ actuators (Fig. 13). While the low-shear regions were between two adjacent actuators for the VGJ actuators, this region extends to the corner vortices for the STJ actuators.

Figure 15 shows the time averaged velocity profiles of the three different operation mode of the SWJ actuators adapted from Ref. [13]. Note that the flow rate to the actuators was slightly different from the current study. Although the jet velocity is usually normalized by a flow related quantity (such as $U_{j e t}$ used in $C_{\mu}$ calculations) and the abscissa by the actuator throat width, we used local maximum to normalize the jet velocity and kept the abscissa dimensional to assess the lateral extent of the actuator's effect. As reported previously [13], the STJ mode produced a jet similar to a turbulent free jet with a peak velocity at the centerline. The velocity profile of the VGJ mode was the same, except that the peak velocity was now shifted off the centerline. Although the profile was shifted, the spanwise extent of the VGJ jet appears to be similar to that of the STJ mode. On the other hand, the oscillatory mode (SWJ) produced a velocity profile with double peaks. The jet half-width of the SWJ actuators were reported to be substantially larger than that of the STJ and VGJ actuators [13], which resulted in increased spanwise coverage.

\section{The Effect of Actuator Placement}

Actuator placement consists of the pitch angle, spreading angle, and the streamwise location of the SWJ actuators. The pitch angle, $\boldsymbol{a}$, is defined as the angle between the jet axis and the surface tangent in the vertical plane. Since the SWJ actuators were installed upstream of the ramp, where the freestream flow was parallel to the surface, the pitch angle is also defined as the angle between the jet axis and the freestream flow. Three different pitch angles, namely $16^{\circ}, 30^{\circ}$, and $45^{\circ}$, were evaluated. Figure 16 shows the effect of the pitch angle on the $C_{p}$ distribution. All other parameters were set to their baseline values. The $C_{p}$ distributions for $\boldsymbol{a}=45^{\circ}$ and $\boldsymbol{a}=30^{\circ}$ are very close to the MVG flow-control case. We observed a slightly better pressure recovery for $\boldsymbol{a}=16^{\circ}$. This may be because the smaller pitch angle results in a more tangential component of the jet blowing. This is also consistent with the previously reported data, where maximum pressure recovery on a backward-facing ramp was obtained using an array of VGJ actuators with $15^{\circ}<a<25^{\circ}$ [8]. Very small (tangential) and large pitch angles were not evaluated in this study. For the cases tested, the $C_{p}$ distribution suggests that small pitch angles are beneficial although the effect is not significant.

The second parameter of the actuator placement is the spreading angle of the SWJ-actuator jet. The spreading angle, $\theta$, is analogous to the skew angle of a conventional VGJ actuator, which is found to be an important parameter in the literature. As reported previously, the jet out of an SWJ actuator oscillates between two actuator exit sidewalls and these periodic oscillations are not sinusoidal $[10,12]$. In fact, the jet spends more time on the actuator sidewalls during the oscillation, which results in double peaks in the mean velocity profiles. As a result, the streamwise vortices are observed on both sides of an SWJ actuator as indicated by clear flow-detachment lines in the flow visualization (Fig. 11). Although the skew angle of the SWJ actuators changes constantly, the spreading angle is defined based on the mean velocity profile. To assess the effect of the spreading or skew angle, two actuators 
with different spreading angles were fabricated. The velocity profile for each actuator was measured at different downstream locations using a hot-wire anemometer on a bench top. Since the flow direction and the hot-wire probe axis were not parallel, pitch corrections were applied to the hot-wire measurements as described in Ref. [12]. The flow rate to the actuators was adjusted to the baseline value. The jet half-widths were obtained using the peak velocity and then the jet half-widths were used to determine the average spreading angle. The spreading angles were found to be approximately $70^{\circ}$ and $110^{\circ}$, respectively. Figure 17 exhibits the time averaged velocity profiles for two different actuators that were measured $1.6 \mathrm{~cm}$ downstream of the actuator throat. Both actuators generated double peak velocity profile. At this particular location, the $\theta=110^{\circ}$ actuator produced almost two times spanwise coverage than that of the $\theta=70^{\circ}$ actuator (jet half-widths were $4.3 \mathrm{~cm}$ and $2.2 \mathrm{~cm}$, respectively)

The effect of the spreading angle on the $C_{p}$ distribution is presented in Fig. 18. While the $\theta=$ $110^{\circ}$ actuator is the baseline case that matches the MVG control case, reducing the spreading angle to $70^{\circ}$ greatly reduces the pressure recovery in the separated flow region. The pressure recovery for both spreading angles appears to be close downstream of $\mathrm{X}=183$ $\mathrm{cm}$, where the centerline flow is fully attached. Since the same actuators with the same flow input were used, the reduced pressure recovery implies reduced actuator efficiency. Reduced efficiency of the SWJ actuators with smaller spreading angle is also consistent with the results about the skew angle of conventional VGJ actuators in the literature. Although there are mixed results about the optimum skew angle of VGJ actuators, an angle between $60^{\circ}$ and $90^{\circ}$ is reported to be well-suited for separation control studies on an APG ramp [8, 25] and variation from $60^{\circ}$ to $90^{\circ}$ in the skew angle does not cause significant changes in the overall flow features [26]. Although we could not test the SWJ actuators with $180^{\circ}$ spreading angle (or $90^{\circ}$ skew angle), for the cases tested, and considering the results provided in the literature about the VGJ-skew angle, a spreading angle between $120^{\circ}$ and $180^{\circ}$ may provide better pressure recovery and thereby may result in better actuator efficiency.

The $C_{p}$ distributions clearly showed the effect of the actuator placement; however, the flow visualization helps to better understand why the smaller spreading angle causes inferior actuator efficiency. The oilflow visualization indicates that the flow control with the $\theta=70^{\circ}$ actuators also keep the flow in the center of the ramp remain attached (Fig. 19). When compared to the baseline case (Fig. 11), the flow-detachment lines are not as clear. This suggests that the generated vortices are not as strong as the baseline case due to the reduced skew (or spreading) angle. In addition, the actuators are effective on a relatively narrow area (about $2.5 \mathrm{~cm}$ wide for each actuator). This is expected because the spreading angle is directly related to the actuator's coverage area. Although the boundary layer is energized and the flow appears to be attached, we observed thicker oilflow patterns beginning from $\mathrm{X}$ $=157 \mathrm{~cm}$ between two adjacent actuators (about $3 \mathrm{~cm}$ wide). The thicker oil patterns indicate a low-shear stress region. These low-shear stress regions and the regions that were influenced by the actuators are distinctly seen side by side downstream of the actuators. This low-shear stress region may be the reason for the reduced pressure recovery for the SWJ actuators with smaller spreading angles. 
Blowing-type flow-control methods are more effective when they are installed close to the flow separation location [8]. In Fig. 18, we also presented the $C_{p}$ distribution with the $\boldsymbol{\theta}=$ $70^{\circ}$ actuator placed at $X_{S W J}=157 \mathrm{~cm}$. Although the smaller spreading actuator provided inferior pressure recovery compared to the baseline case, moving the actuators closer to the separation location alleviated the inefficacy and provided similar pressure recovery to that of the baseline case. If we look closely in Fig. 18, we see an offset in the $C_{p}$ distribution between $X=162 \mathrm{~cm}$ and $X=168 \mathrm{~cm}$. This region corresponds to the separated flow region as indicated by stagnant oil flow between two adjacent actuators in Fig. 20, which shows the surface oilflow visualization of the $\theta=70^{\circ}$ actuators placed at $X_{S W J}=157 \mathrm{~cm}$. The smaller spreading angle resulted in smaller spanwise coverage; therefore, the actuators could not eliminate the flow separation in these regions. Similar flow separation was also observed in a previous study [13] and these separation regions persist even when doubling the momentum coefficient. Figure 21 presents the surface oilflow visualization of the $\theta=110^{\circ}$ actuator placed close to the separation location $\left(X_{S W J}=157 \mathrm{~cm}\right)$ but with half the momentum coefficient $\left(C_{\mu}=0.2 \%\right)$. As shown by the surface flow visualization, the generated streamwise vortices maintained attached flow even with $C_{\mu}$ reduced by $50 \%$. Another noticeable difference is the clear flow-detachment lines in the APG region for the downstream actuator that were not observed in the APG region for the baseline (upstream actuator) case. This suggests that the vortices generated by the upstream actuator have reduced circulation level by the time they reach the APG region. When compared to Fig. 20, where the only difference is the spreading angle, the $\theta=110^{\circ}$ actuators were able to eliminate the separated flow region between two adjacent actuators even with half the momentum coefficient. In addition, we see clear flow detachment lines, which were not present in Fig. 20, indicating that the vortex generated with the $\theta=110^{\circ}$ actuator is stronger than that of the $\theta=70^{\circ}$ actuator.

As is consistent with the flow visualization figures, the $C_{p}$ distributions for two actuator locations $\left(X_{S W J}=147 \mathrm{~cm}\right.$ and $\left.X_{S W J}=157 \mathrm{~cm}\right)$ also confirms that moving the actuator closer to the separation location greatly benefits the flow control authority. As shown in Fig. 22, the actuators at $X_{S W J}=157 \mathrm{~cm}$ provided more pressure recovery than the reference flow control (MVG) case. Since $C_{\mu}$ is fixed, more pressure recovery directly indicates a more efficient actuator. After $\mathrm{X}=180 \mathrm{~cm}$, the $C_{p}$ distributions become similar due to the attached flow. The effect is more pronounced near $X=168 \mathrm{~cm}$ due to the steeper APG. Note that the pitch angle of the downstream $\left(X_{S W J}=157 \mathrm{~cm}\right)$ actuator was larger $\left(\boldsymbol{a}=42^{\circ}\right)$ than the baseline actuator $\left(\boldsymbol{a}=30^{\circ}\right)$ due to the ramp surface. However, as discussed in the previous section, the effect of the pitch angle was negligible, especially for $\boldsymbol{a}=30^{\circ}$ and $\boldsymbol{a}=45^{\circ}$. The reason for this increased performance may be that the streamwise vortices generated by the downstream $\left(X_{S W J}=157 \mathrm{~cm}\right)$ actuators still have higher circulation by the time they reach the separated flow region due to their proximity. On the other hand, the vortices generated by the upstream actuators $\left(X_{S W J}=147 \mathrm{~cm}\right)$ become more diffuse as they travel downstream and by the time they reach the APG region, they do not have enough circulation to energize the boundary layer. 


\section{E. The Effect of Actuator Size}

The effect of the actuator size on the actuator performance was studied using three different sizes of SWJ actuators. Actuator size is important because it is directly related to the actuators' power/mass consumption. In this study, the different sizes of actuators were fabricated by scaling down the baseline actuator by $60 \%(0.6 \mathrm{X})$ and $30 \%(0.3 \mathrm{X})$, respectively, without changing the actuator aspect ratio, spacing, or its streamwise location. All other parameters were set to their baseline values. Comparison of the $C_{p}$ distributions for the actuators with different scales indicates substantial pressure recovery (Fig. 23) for all cases. The baseline configuration provided slightly better pressure recovery, and both of the scaled-down actuators produced identical pressure distributions. Although the required flow rate reduces linearly with the actuator scale, the required pressure to supply the same $C_{\mu}$ increases proportionally for small-scale actuators (Table 2). We also evaluated the actuator performance for a fixed $C_{\pi}=0.8 \%$ because the actuator modification requires different power/mass to operate them. As shown in Fig. 24, the baseline actuator provided the most pressure recovery, and the smallest sized actuator provided the least. This may be because $C_{\mu}$ gradually reduces (Table 3 ) as the actuator size reduces thereby providing less pressure recovery compared to the fixed- $C_{\mu}$ data in Fig. 23.

In order to see the global effect of the actuator size on the flow field, we performed surface flow visualization. Figure 25 shows the flow visualization results obtained using the $0.6 \mathrm{X}$ actuators with the same momentum coefficient $\left(C_{\mu}=0.4 \%\right)$. Consistent with the $C_{p}$ distribution, we obtain very similar flow patterns to the baseline case (Fig. 11). The vortices produced by the SWJ actuators traveled downstream and generated distinct flow-detachment lines. These flow-detachment lines appear to merge at $X=156 \mathrm{~cm}$ similar to the baseline case. The generated vortices energized the boundary layer and allowed the flow in the center of the ramp to remain attached. The lateral extent of the actuator, i.e., the jet spreading, appears to be similar to the baseline case (Fig. 11). Figure 26 presents the flow visualization for the smallest-sized $(0.3 \mathrm{X})$ actuators with the same $\mathrm{C}_{\mu}$. Although the $C_{p}$ distributions indicated similar performance to the large-scale actuators, the oilflow visualization revealed some differences. The oilflow patterns near the tunnel centerline indicate attached flow primarily due to the middle actuator, which was located at the tunnel centerline. Therefore, the $C_{p}$ distribution of the smallest sized actuator was very similar to the large-scale actuators (Fig. 23). The flow visualization exhibits low-shear regions from $X=157 \mathrm{~cm}$ to $X=165 \mathrm{~cm}$ due to the thicker boundary layer between two adjacent actuators (about $3 \mathrm{~cm}$ wide area). Similar low-shear regions were also observed in the flow-visualization figures of the VGJ actuators (Fig. 13) and the SWJ actuators with small spreading angles (Fig. 19). These lowshear regions between two adjacent actuators indicate areas where the actuators may not be able to energize the local boundary layer. As shown at the top of the figures, the lateral extent of the actuators, i.e., the jet spreading, is limited compared to the baseline or $0.6 \mathrm{X}$ actuators. The actuators act on about a $2.5 \mathrm{~cm}$ wide area downstream of each actuator; therefore, more actuators are needed between two actuators to energize the boundary layer and hence to eliminate pressure losses. In addition, compared to the baseline or $0.6 \mathrm{X}$ actuators, the flow-detachment lines generated by the counter-rotating vortices are not very clear. Although a clear flow detachment line indicates stronger vortex for similar configurations (such as Fig. 11 vs. Fig. 19 and Fig. 21 vs. Fig. 20), it does not necessarily 
indicate a weaker vortex for two different configurations. As shown in Table 2, the $V R$ of the $0.3 \mathrm{X}$ is much higher than the large scale actuators and according to Rixon et al. [27], higher $V R$ jets produce stronger vortices but also result in deeper jet penetration. Therefore the streamwise vortices generated by the $0.3 \mathrm{X}$ actuator are not weaker, but are farther away from the surface compared to the large scale actuator.

As shown by the time averaged jet velocity measurements (Fig. 27), the velocity profiles of $1 \mathrm{X}$ and $0.6 \mathrm{X}$ actuators have similar lateral extent, which is consistent with the $C_{p}$ distributions as well as the oilflow visualization, where both actuator configurations produced similar performance. Although the $C_{p}$ distribution showed similar pressure recovery for the smallest-sized actuator (in Fig. 24), the oilflow visualization indicated that the $0.3 \mathrm{X}$ actuator is effective only in a limited spanwise region. The velocity profile of the $0.3 \mathrm{X}$ actuator confirms this finding such that the actuator produces a relatively narrow jet. When we consider the spreading angles of different sized actuators, we find out that while the spreading angle of the $0.6 \mathrm{X}$ actuator (approximately $\theta=100^{\circ}$ ) is close to that of the $1 \mathrm{X}$ actuator, it is considerably less (approximately $\theta=60^{\circ}$ ) for the $0.3 \mathrm{X}$ actuator, which may be the reason for its reduced performance.

\section{Conclusion}

An experimental study was performed to investigate the effect of different actuator parameters on the performance of sweeping jet actuators in controlling flow separation on an adverse pressure gradient ramp model. The parameters investigated were actuator operation mode, actuator placement, and actuator size. Surface pressure measurements and surface oilflow visualization were used to determine the effects of each parameter on the actuator performance. For select cases, 2D PIV measurements of the ramp flow and hot-wire measurements of the actuator's jet flow were also obtained. An array of MVGs was used on the ramp as a passive flow control device, and the resultant $C_{p}$ distribution was used as a target $C_{p}$ distribution to be achieved by the SWJ actuators. The target $C_{p}$ distribution was achieved and attached flow was maintained with the baseline SWJ configuration. The effect of each parameter was characterized by comparing the pressure distribution and the surfaceflow patterns to that of the baseline case.

First, the SWJ actuators were compared to two other well-known active flow-control methods, namely straight jets and vortex generating jets with the same configurations. These three different blowing methods were obtained by operating the same actuators with different modes. The $C_{p}$ measurements and the surface oilflow visualization indicated that the STJ actuators are the least effective because they energize the boundary layer only by adding momentum. On the other hand, the VGJ and SWJ actuators generate streamwise vortices that provide more pressure recovery by distributing the high-momentum fluid in the boundary layer through mixing. The superiority of the SWJ actuators over the VGJ actuators was attributed to the unsteady (spatially oscillating) streamwise vortices generated by the SWJ actuators affecting on both sides of the actuators, whereas the VGJ actuators generated steady streamwise vortices only on one side. These unsteady and spatially oscillating vortices resulted in increased coverage, more pressure recovery, and reduced flow separation. The flow visualization of the VGJ actuators exhibited local low-shear regions 
between two adjacent VGJ actuators, where the generated streamwise vortices were unable to energize the boundary layer. Therefore, in order to recover the pressure losses between two adjacent actuators, more actuators are needed in the spanwise plane.

The effect of actuator placement was studied by varying the pitch angle, spreading angle, and streamwise location of the SWJ actuators. Since the actuator placement is not related to the actuator mass flow input, an increase in the actuator performance directly implies an increased actuator efficiency. Although the effect of the actuator pitch angle is small on the actuator performance, the spreading angle is found to be an important parameter and reducing the spreading angle greatly reduces the pressure recovery thereby reducing the actuator efficiency. The effect of the spreading angle is twofold. First, the spreading angle is directly related to the actuator spanwise coverage; therefore, the actuators with small spreading angles require more actuators or flow rate to achieve similar performance. Second, the spreading angle is also related to the skew angle of a jet described in the literature. As such, we would expect an actuator with a small spreading angle to generate vortices with low circulation levels and actuators with large spreading angles to generate vortices with high circulation levels. By investigating the actuator performance at two streamwise locations, we observed that the actuator performs better when placed closer to the flow separation location. This is because the generated vortices have higher circulation when they reach the separated flow region. On the other hand, the strength of the vortices generated by the upstream actuators decays as they travel downstream and at the APG region, they do not have enough circulation to energize the boundary layer.

The effect of actuator size was studied by varying the actuator scale while keeping the aspect ratio the same. Since the actuators with different sizes require different pressures and flow rates to maintain the same momentum coefficient, the results were provided for a fixed momentum coefficient as well as for a fixed power coefficient. Although the different sized $(1 \mathrm{X}, 0.6 \mathrm{X}$, and $0.3 \mathrm{X})$ actuators provided similar centerline pressure recovery, the flow visualization indicated that the smallest-sized actuators $(0.3 \mathrm{X})$ have limited spanwise coverage and are unable to energize the boundary layer between two adjacent actuators. Hotwire measurement of the actuators on a benchtop confirms that the $0.3 \mathrm{X}$ actuators have different behavior than the other actuators. Although the time averaged velocity profiles of the $1 \mathrm{X}$ and $0.6 \mathrm{X}$ actuators are similar (and hence their effects), the velocity profiles of the smallest $(0.3 \mathrm{X})$ actuators are much narrower resulting in considerably reduced jet spreading, which may be the cause of the reduced performance. The reduction of the jet spreading at the small scales is currently unknown and left as a future work.

\section{Acknowledgments}

The author would like to thank the NASA Fixed Wing Project for funding this research and the following individuals for their support: Catherine McGinley, Luther Jenkins, John Lin, Latunia Melton, and Charlie Debro.

\section{Nomenclature}
$\mathbf{A}_{\text {ref }}$
reference area $\left(\mathrm{m}^{2}\right)$
$\mathbf{A}_{\text {jet }}$
total area of actuator throats $\left(\mathrm{m}^{2}\right)$ 


$\begin{array}{ll}\mathbf{C}_{\boldsymbol{\mu}} & \text { momentum coefficient } \\ \mathbf{C}_{\boldsymbol{\pi}} & \text { power coefficient } \\ \mathbf{C}_{\mathbf{p}} & \text { pressure coefficient } \\ \mathbf{f} & \text { oscillation frequency }(\mathrm{Hz} \\ \mathbf{P}_{\mathbf{a c t}} & \text { actuator plenum pressure }(\mathrm{kPa}) \\ \mathbf{Q} & \text { actuator volume flow rate }(\mathrm{L} / \mathrm{s}) \\ \mathbf{R x y} & \text { nondimensional turbulent shear stress } \\ \mathbf{U}_{\boldsymbol{\infty}} & \text { freestream velocity (m/s) } \\ \mathbf{X}_{\mathbf{S W J}} & \text { actuator-streamwise location (cm) } \\ \mathbf{V R} & \text { velocity ratio } \\ \mathbf{a} & \text { pitch angle } \\ \delta & \text { boundary-layer thickness (mm) } \\ \boldsymbol{\rho}_{\boldsymbol{O}} & \text { freestream air density } \\ \boldsymbol{\theta} & \text { spreading angle } \\ \overline{\omega_{z}} & \text { nondimensional spanwise vorticity }\end{array}$

\section{References}

[1]. Bezos-O'Connor GM, Mangelsdorf MF, Maliska HA, "Fuel Efficiencies through Airframe Improvements," AIAA Paper 2011-3530, 62011.

[2]. Gad-el-Hak M, and Bushnell DM, "Separation Control: Review," Journal of Fluids Engineering, Vol. 113, No. 1, 1991, pp. 5-30.

[3]. Schubauer GB, and Spangenberg W, "Forced Mixing in Boundary Layers," Journal of Fluid Mechanics, Vol. 8, No. 01, 1960, pp. 10-32.

[4]. Jenkins L, Gorton SA, and Anders S, "Flow Control Device Evaluation for an Internal Flow with an Adverse Pressure Gradient," AIAA Paper 2002-0266, 12002.

[5]. Lin J, Howard F, and Selby G, "Small-Submerged Vortex Generators for a Turbulent Flow Separation Control," Journal of Spacecraft and Rockets, Vol. 27, No. 5, 1990, pp. 503-507.

[6]. McCormick D, "Shock-Boundary Layer Interaction Control with Low-Profile Vortex Generators and Passive Cavity,” AIAA Paper 1992-0064, 11992.

[7]. Lin JC, "Review of Research on Low-Profile Vortex Generators to Control Boundary-Layer Separation,” Progress in Aerospace Sciences, Vol. 38, No. 4, 2002, pp. 389-420.

[8]. Selby G, Lin J, and Howard F, "Control of Low-Speed Turbulent Separated Flow Using Jet Vortex Generators,” Experiments in Fluids, Vol. 12, No. 6, 1992, pp. 394-400.

[9]. Gregory JW, and Tomac MN, “A Review of Fluidic Oscillator Development,” AIAA Paper 20132474, 62013.

[10]. Vatsa V, Koklu M, Wygnanski I, "Numerical Simulation of Fluidic Actuators for Flow Control Applications," AIAA Paper 2012-3239, 62012.

[11]. Ostermann F, Woszidlo R, Nayeri C, "Experimental Comparison between the Flow Field of Two Common Fluidic Oscillator Designs," AIAA Paper 2015-0781, 12015. 
[12]. Koklu M, and Melton LP, "Sweeping Jet Actuator in a Quiescent Environment," AIAA Paper 2013-2477, 62013.

[13]. Koklu M, and Owens LR, "Flow Separation Control Over a Ramp Using Sweeping Jet Actuators," AIAA Paper 2014-2367, 62014.

[14]. Melton LP, “Active Flow Separation Control on a NACA 0015 Wing Using Fluidic Actuators," AIAA Paper 2014-2364, 62014.

[15]. Seele R, Tewes P, Woszidlo R, "Discrete Sweeping Jets as Tools for Improving the Performance of the V-22," Journal of Aircraft, Vol. 46, No. 6, 2009, pp. 2098-2106.

[16]. Cerretelli C, Wuerz W, and Gharaibah E, "Unsteady Separation Control on Wind Turbine Blades using Fluidic Oscillators," AIAA Journal, Vol. 48, No. 7, 2010, pp. 1302-1311.

[17]. Seifert A, Stalnov O, Sperber D, "Large Trucks Drag Reduction Using Active Flow Control," AIAA Paper 2008-0743, 12008.

[18]. Woszidlo R, Stumper T, Nayeri C, "Experimental Study on Bluff Body Drag Reduction with Fluidic Oscillators,” AIAA Paper 2014-0403, 12014.

[19]. Andino MY, Lin JC, Washburn AE, "Flow Separation Control on a Full-Scale Vertical Tail Model using Sweeping Jet Actuators," AIAA Paper 2015-0785, 12015.

[20]. Scarano F, and Riethmuller M, “Advances in Iterative Multigrid PIV Image Processing," Experiments in Fluids, Vol. 29, No. 1, 2000, pp. S051-S060.

[21]. Kumar V, "Separation Control in Adverse Pressure Gradient Using High-Speed Microjets," Ph.D. Dissertation, Mechanical Engineering, Florida State Univ., Tallahassee, FL, 2008.

[22]. Seele R, Graff E, Lin J, "Performance Enhancement of a Vertical Tail Model with Sweeping Jet Actuators," AIAA Paper 2013-0411, 12013.

[23]. Koklu M, "Effect of a Coanda Extension on the Performance of a Sweeping-Jet Actuator", AIAA Journal, Vol. 54, No. 3, 2016, pp.1131-1134.

[24]. König B, Fares E, and Nölting S, "Fully-Resolved Lattice-Boltzmann Simulation of Vane-Type Vortex Generators," AIAA Paper 2014-2795, 62014.

[25]. Stillfried FV, Wallin S, and Johansson AV, "Evaluation of a Vortex Generator Model in Adverse Pressure Gradient Boundary Layers," AIAA Journal, Vol. 49, No. 5, 2011, pp. 982-993.

[26]. Zhang X, "Co-and Contrarotating Streamwise Vortices in a Turbulent Boundary Layer," Journal of Aircraft, Vol. 32, No. 5, 1995, pp. 1095-1101.

[27]. Rixon GS, and Johari H, "Development of a Steady Vortex Generator Jet in a Turbulent Boundary Layer," Journal of Fluids Engineering, Vol. 125, 2003, pp. 1006-1015. 


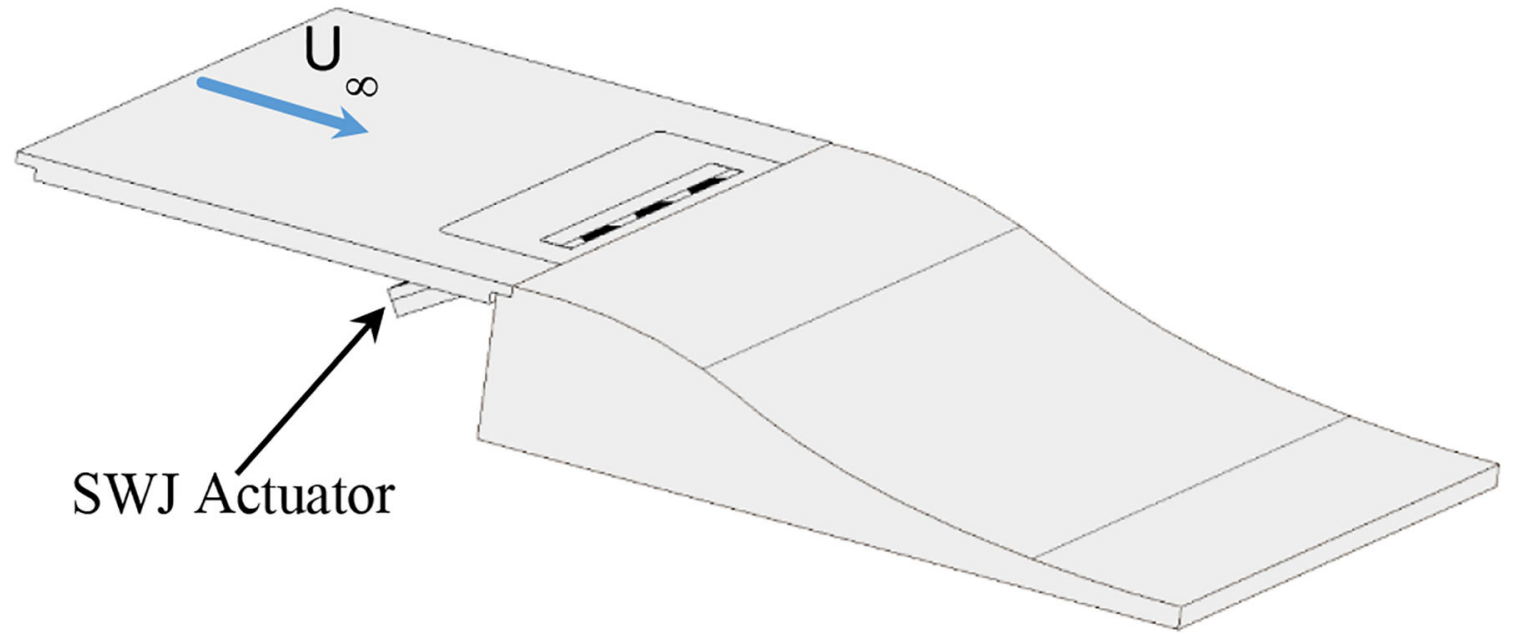

Fig. 1.

Schematic of the APG ramp in the tunnel. 


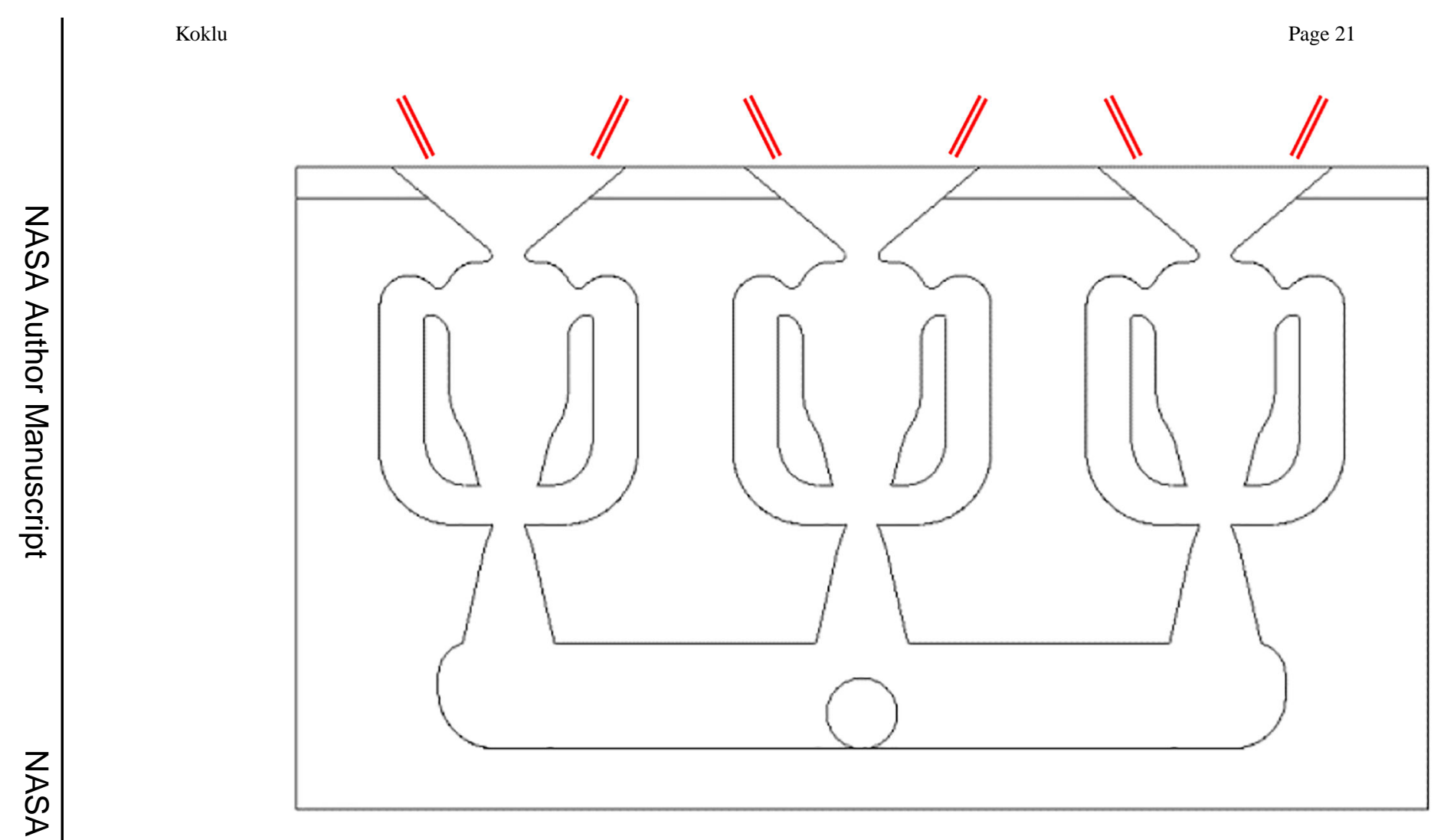

Fig. 2.

SWJ actuators and the spanwise distribution of MVGs relative to SWJ exits. 


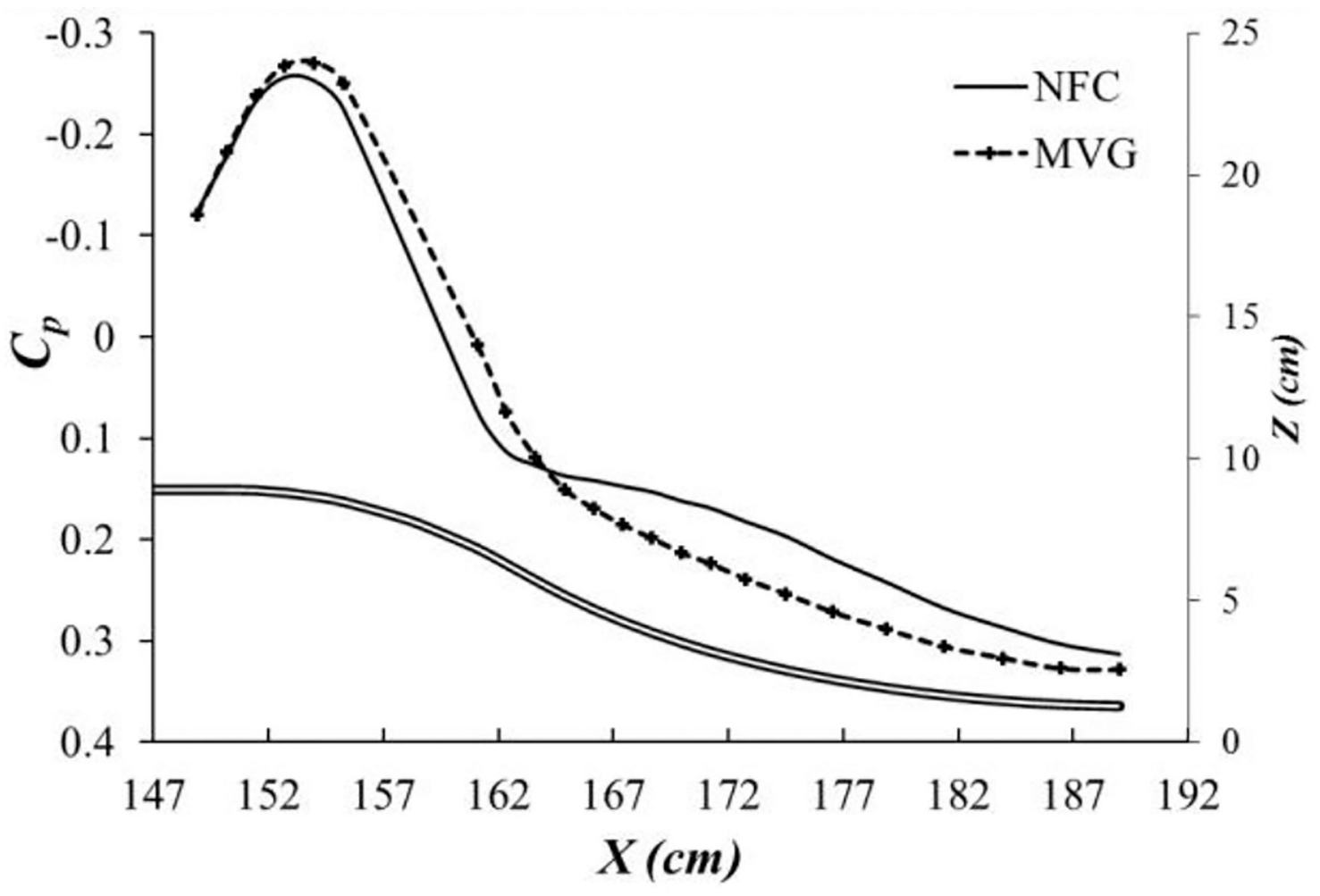

Fig. 3.

Centerline $C_{p}$ distribution along the APG ramp for NFC and MVG cases. 


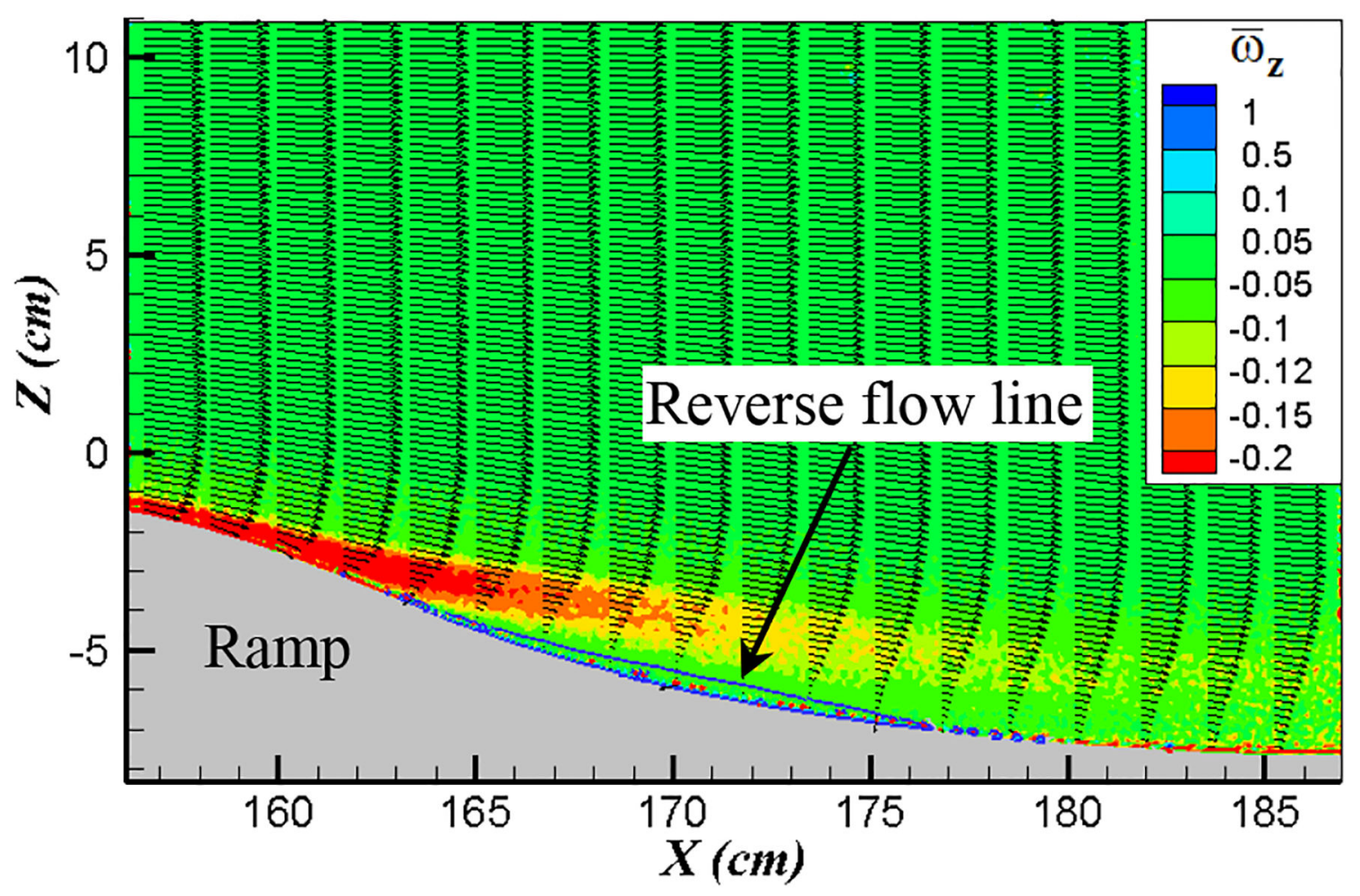

Fig. 4.

Contours of spanwise vorticity showing the separated flow over the APG ramp (NFC case). 


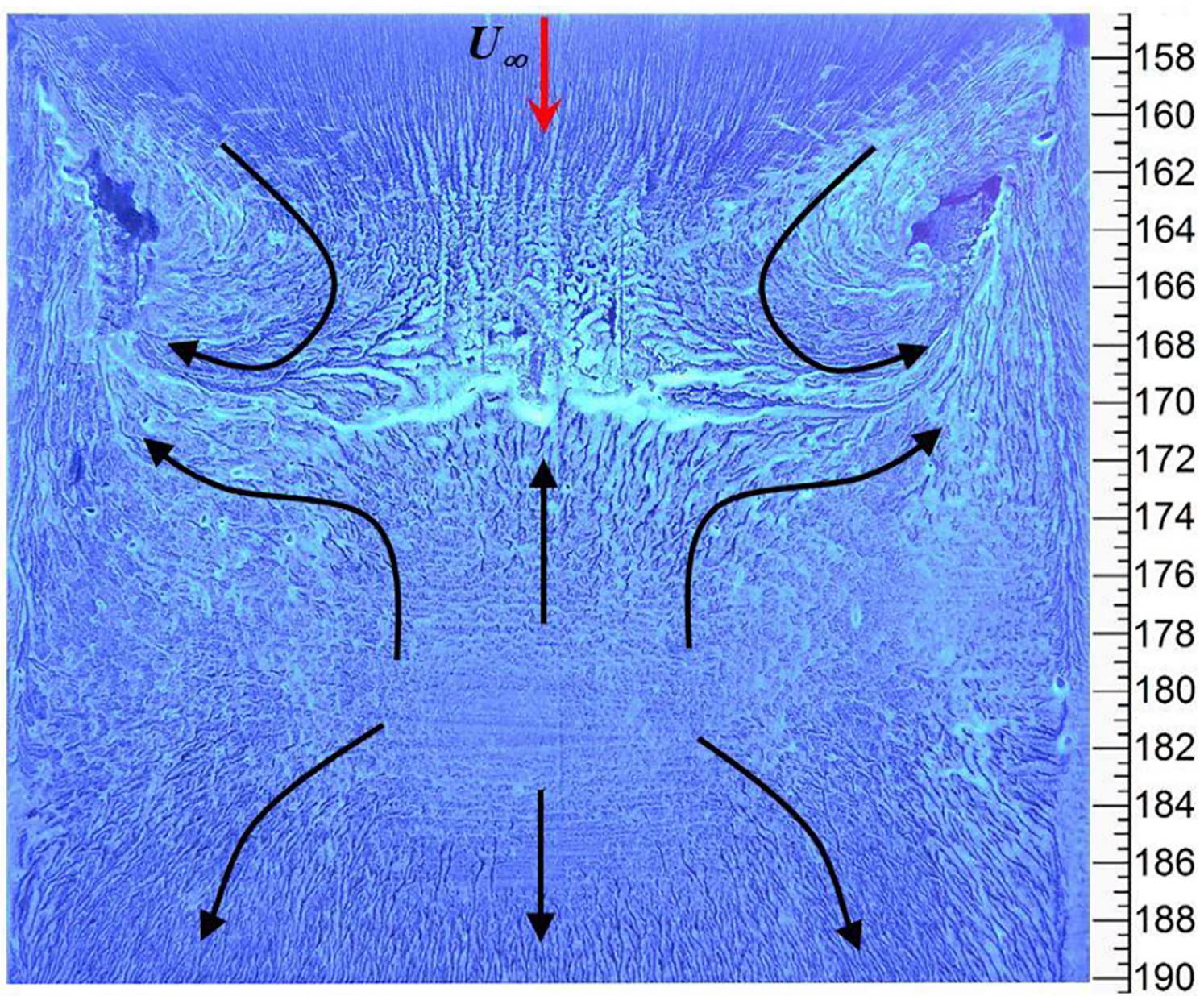

Fig. 5.

Surface oilflow visualization of separated flow over the APG ramp (NFC). 






Fig. 6.

Surface oilflow visualization of flow control with MVGs.






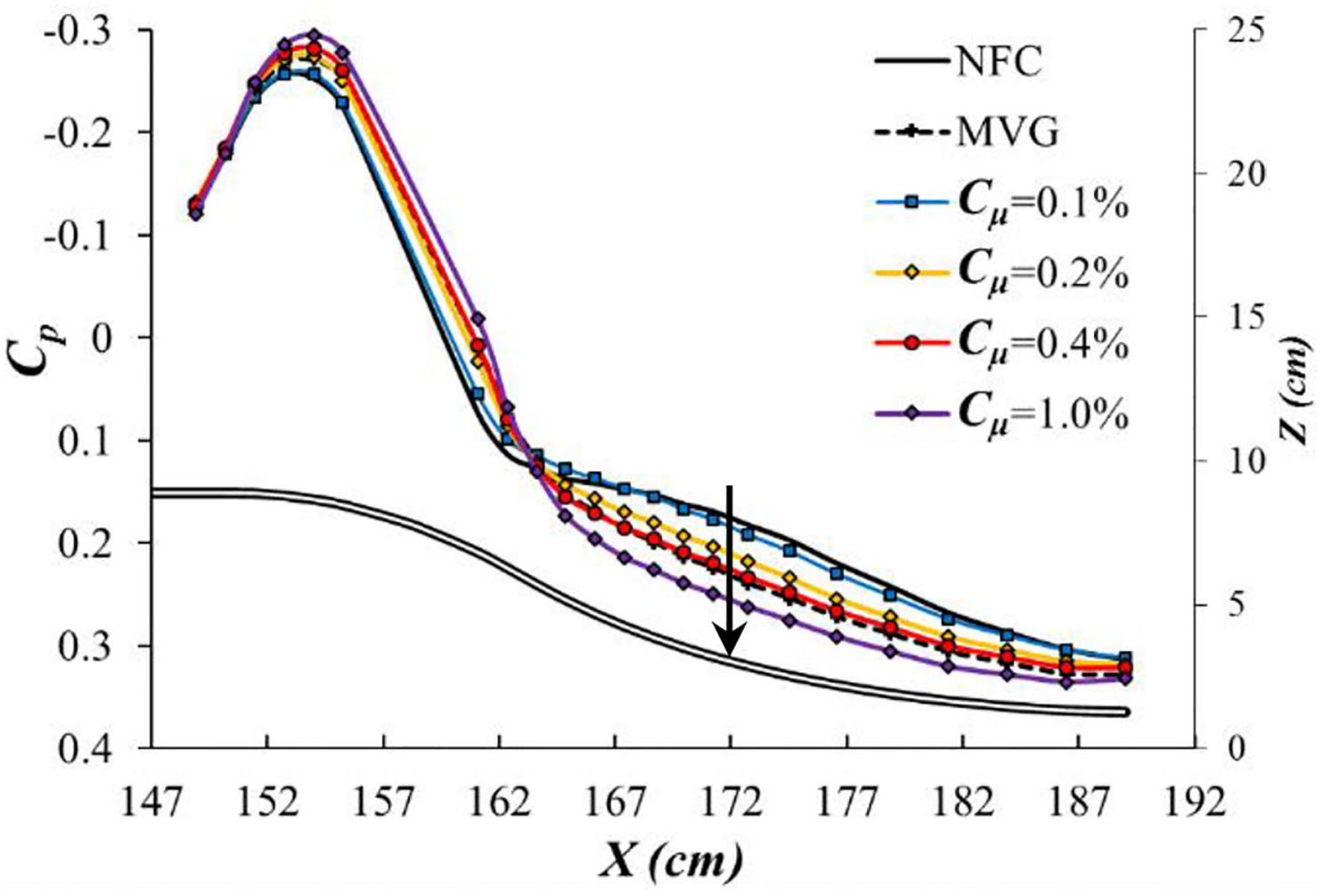

Fig. 7.

The effect of $C_{\mu}$ on the $C_{p}$ distribution. 


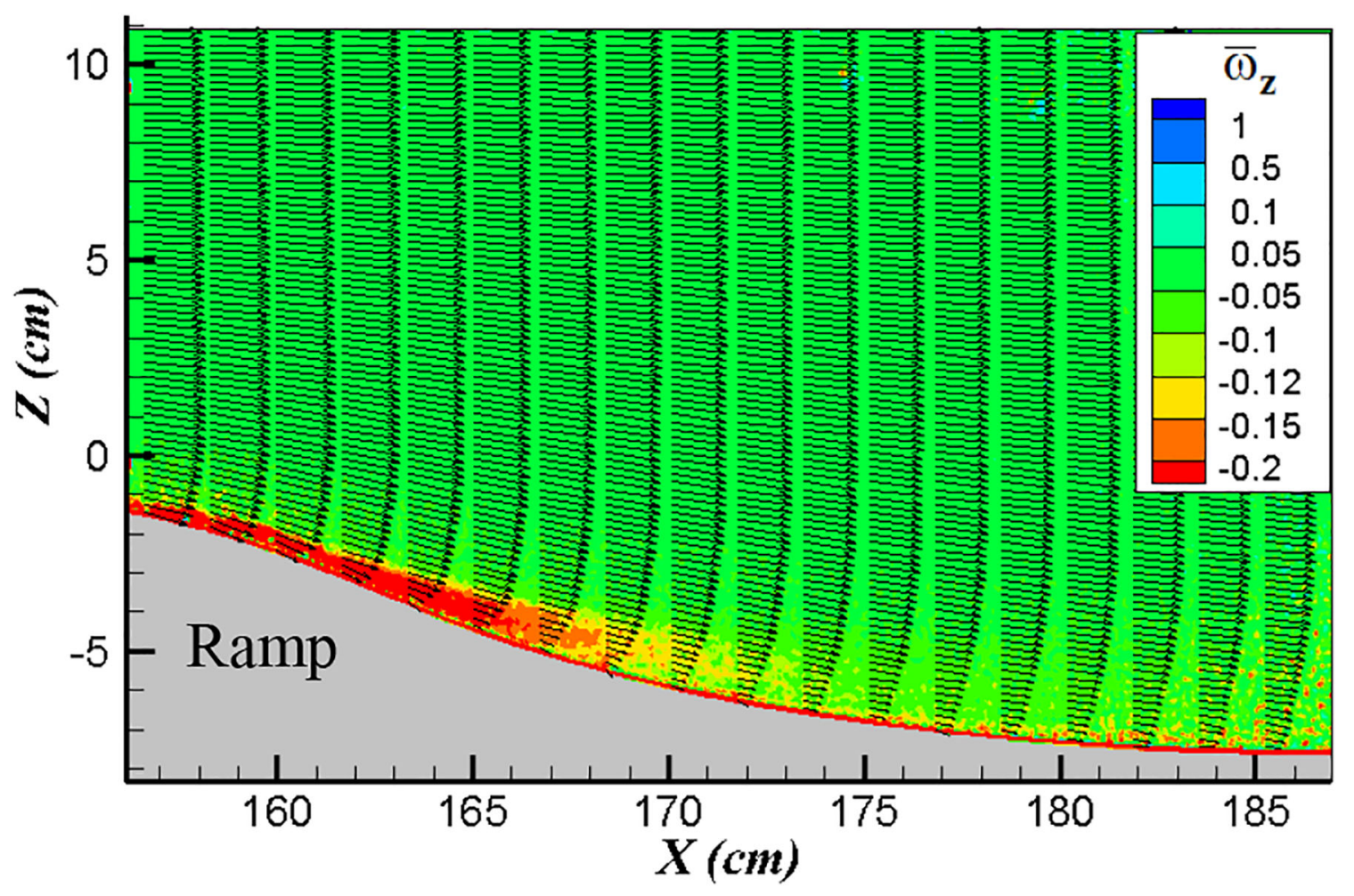

Fig. 8.

Contours of spanwise vorticity and in-plane velocity vectors for baseline case. 

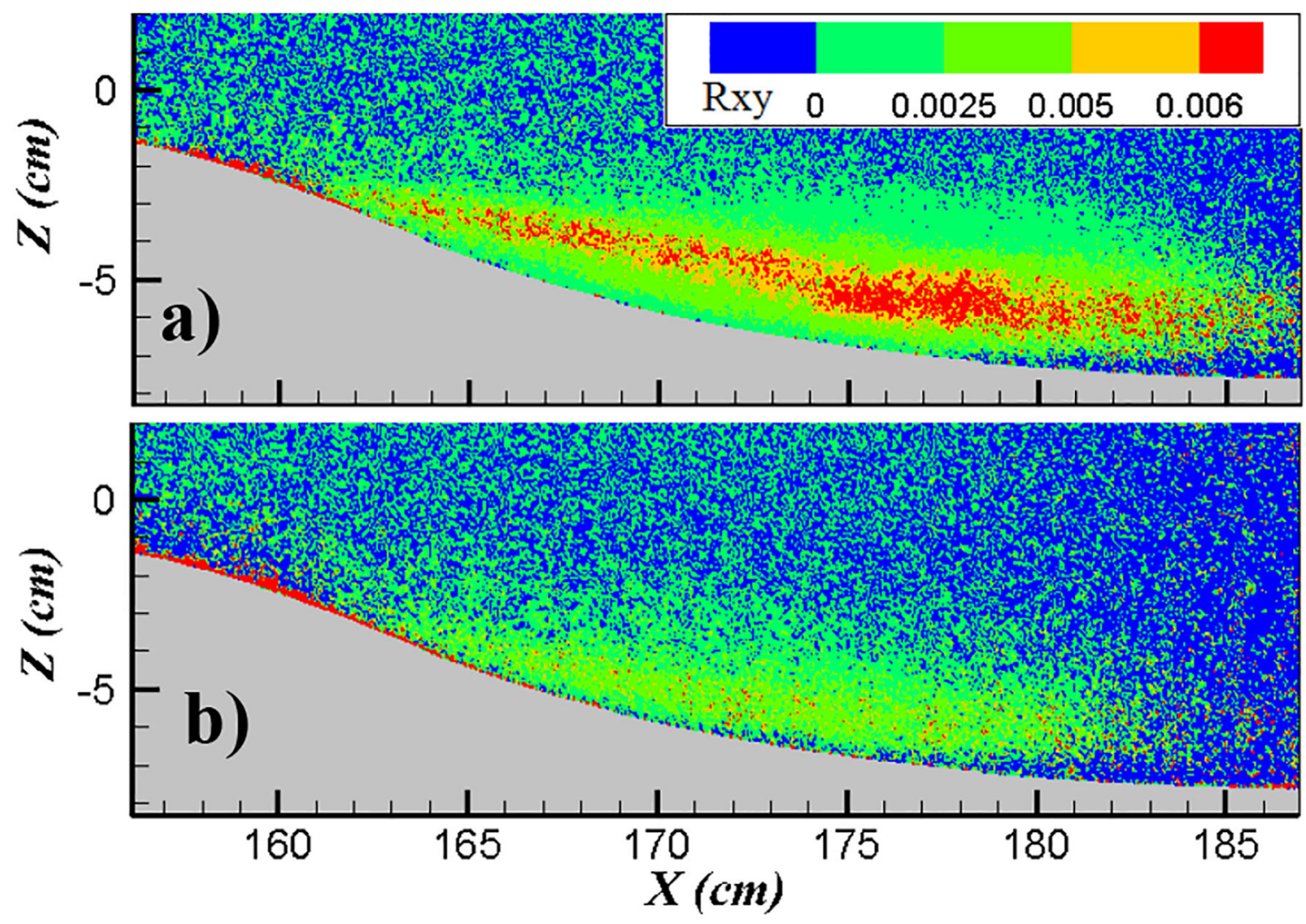

Fig. 9.

Contours of turbulence shear stress for a) separated flow, b) baseline flow control. 




Fig. 10.

Comparison of velocity profiles at different streamwise stations. 


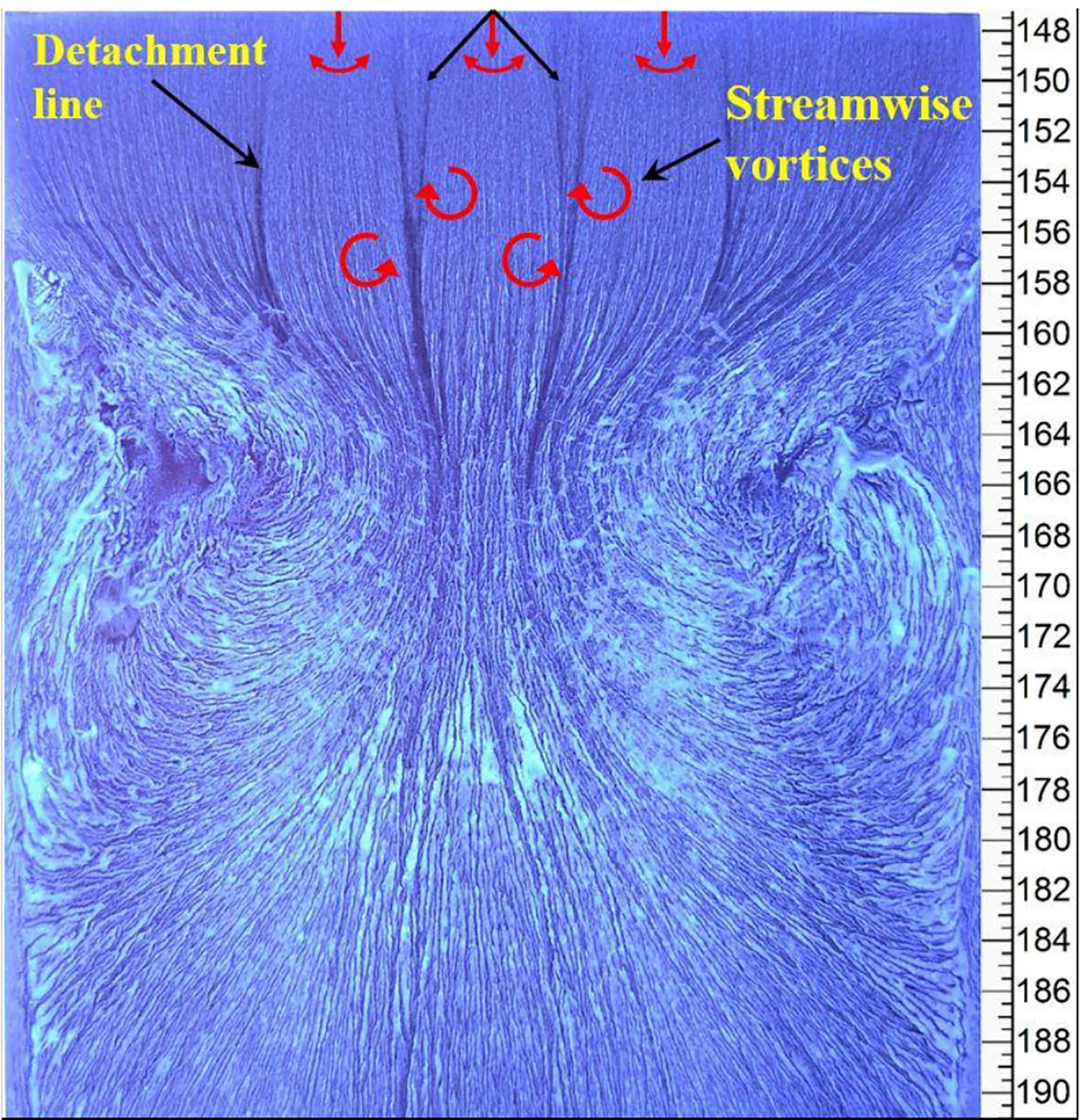

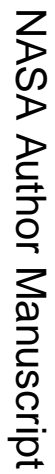

Fig. 11.

Oilflow visualization of the baseline case. 



Fig. 12.

The effect of operation modes on the $C_{p}$ distribution a) $C_{\mu}=0.4 \%$, b) $C_{\pi}=0.8 \%$. 


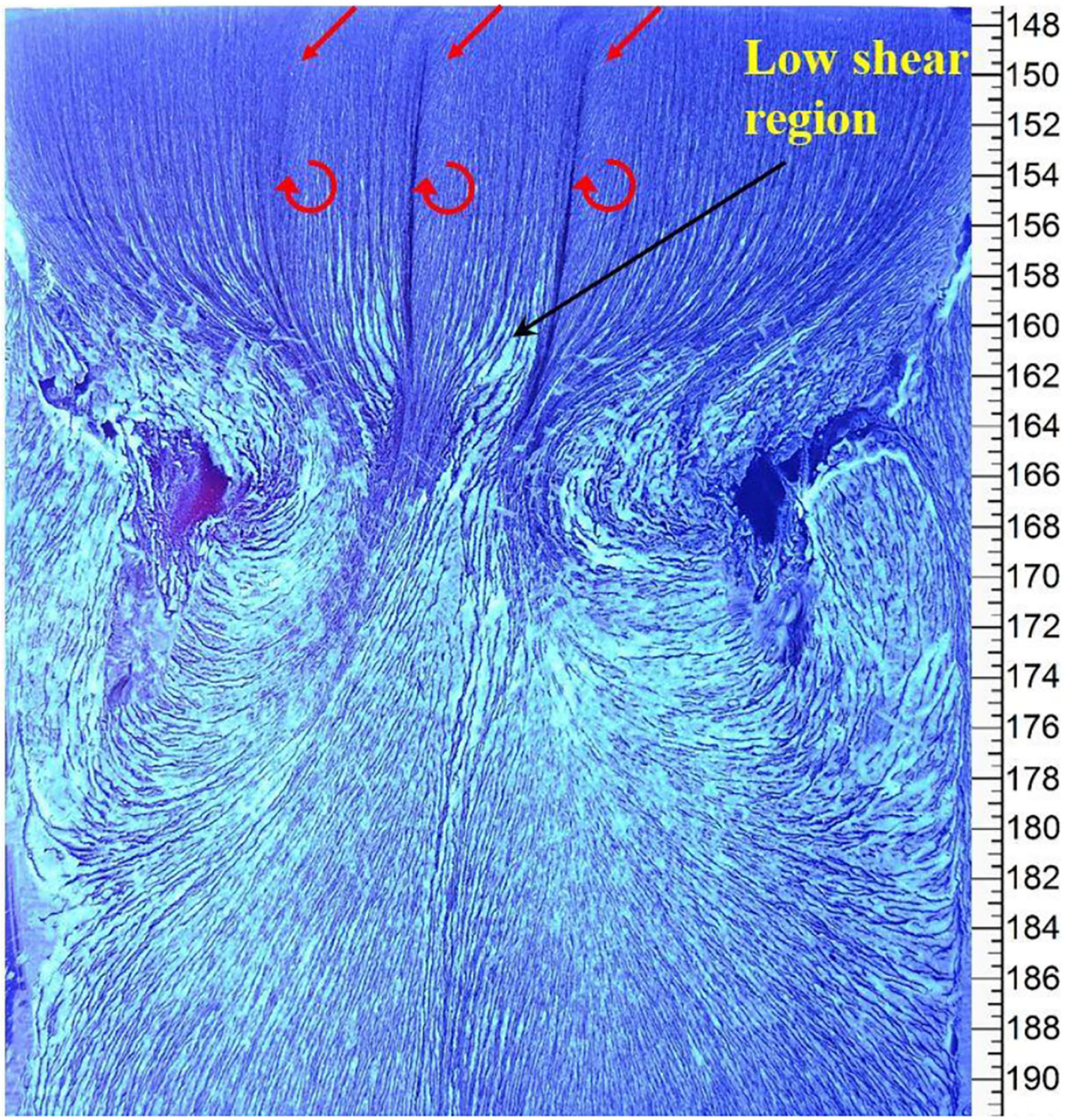

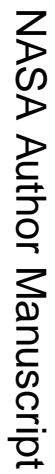

Fig. 13.

Oilflow visualization for the VGJ mode. 


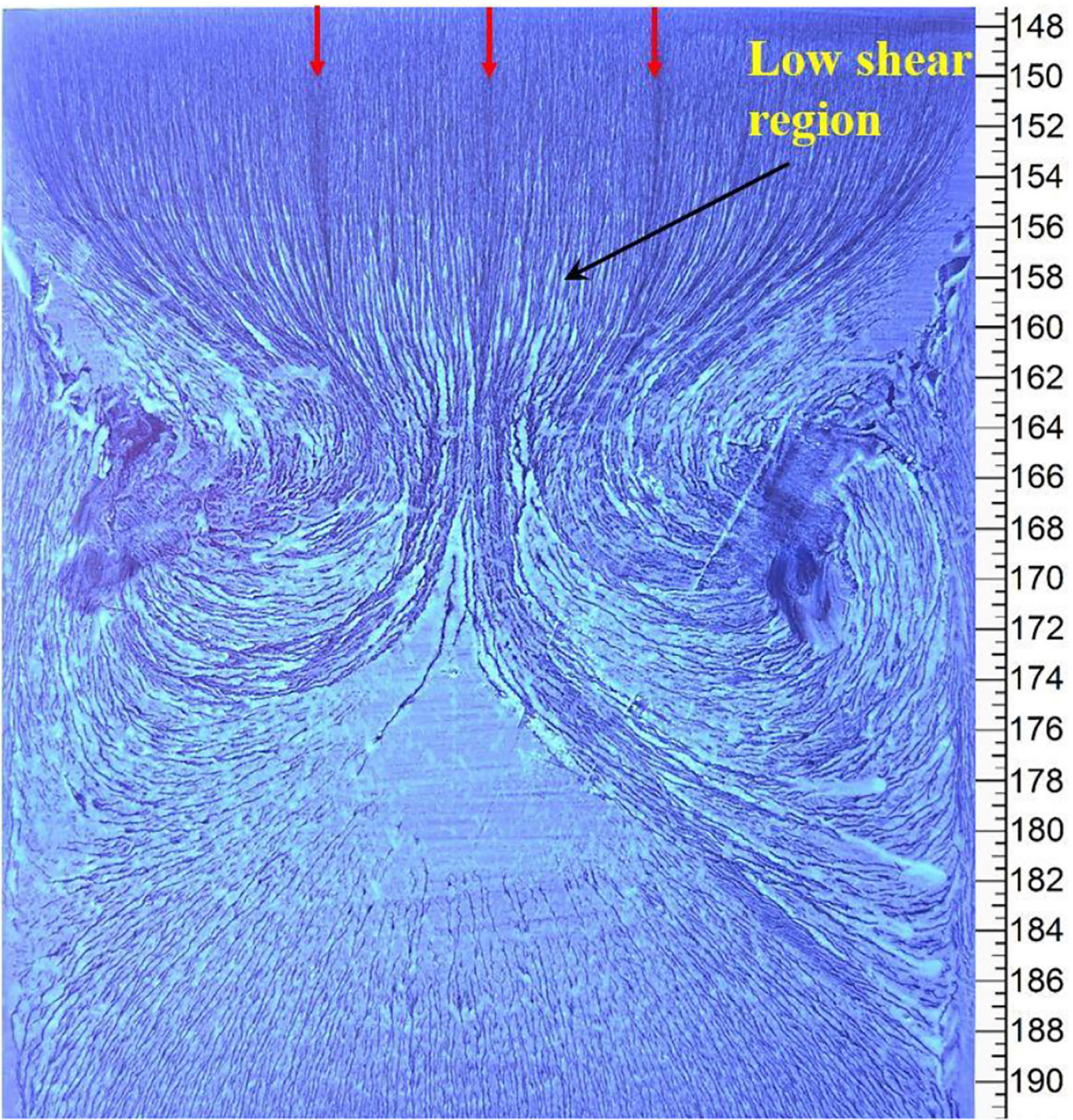

Fig. 14.

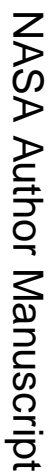

Oilflow visualization for the STJ mode. 


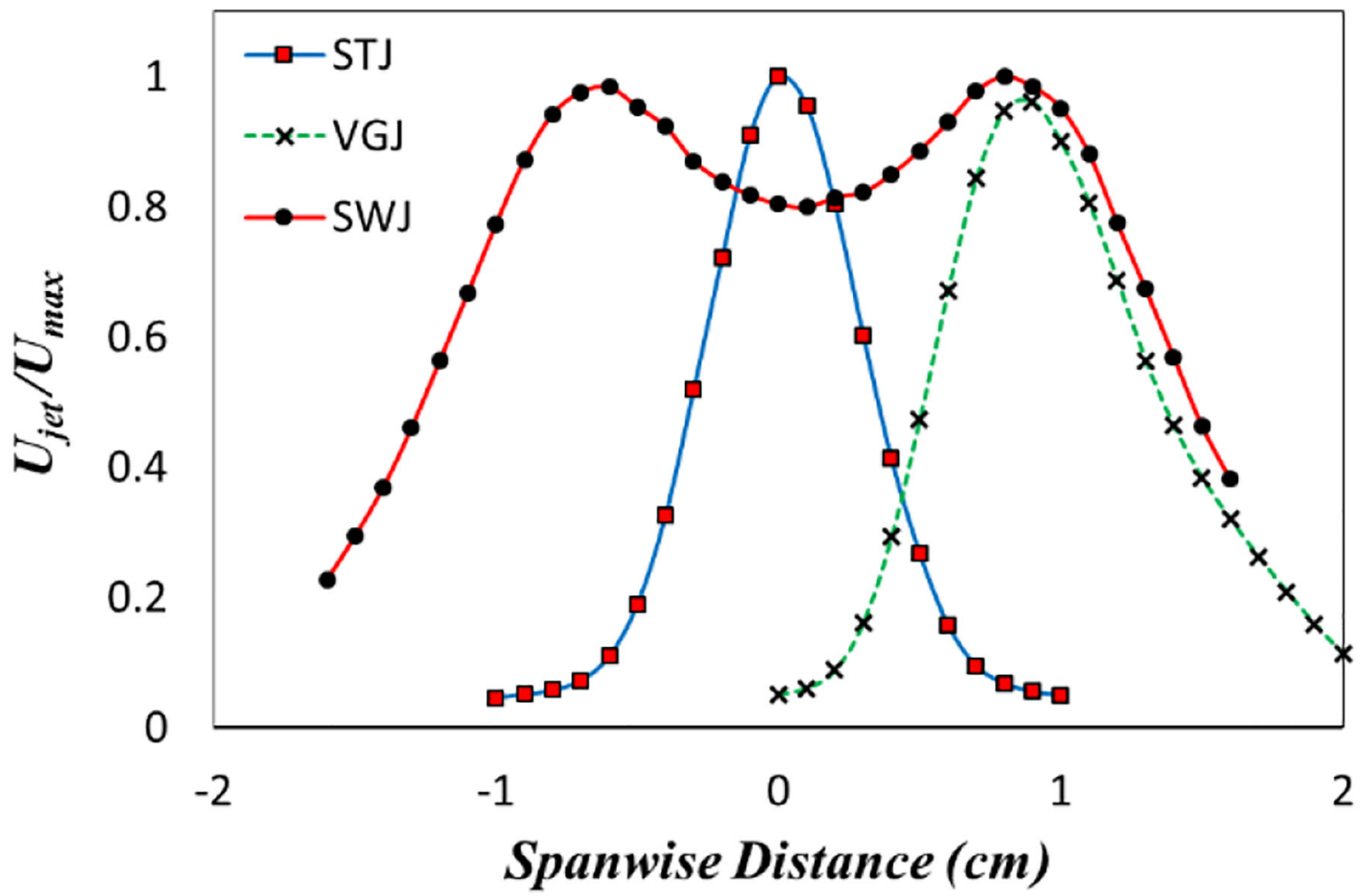

Fig. 15.

Time averaged jet velocity profiles with different operation modes (adapted from Ref. [13]) 


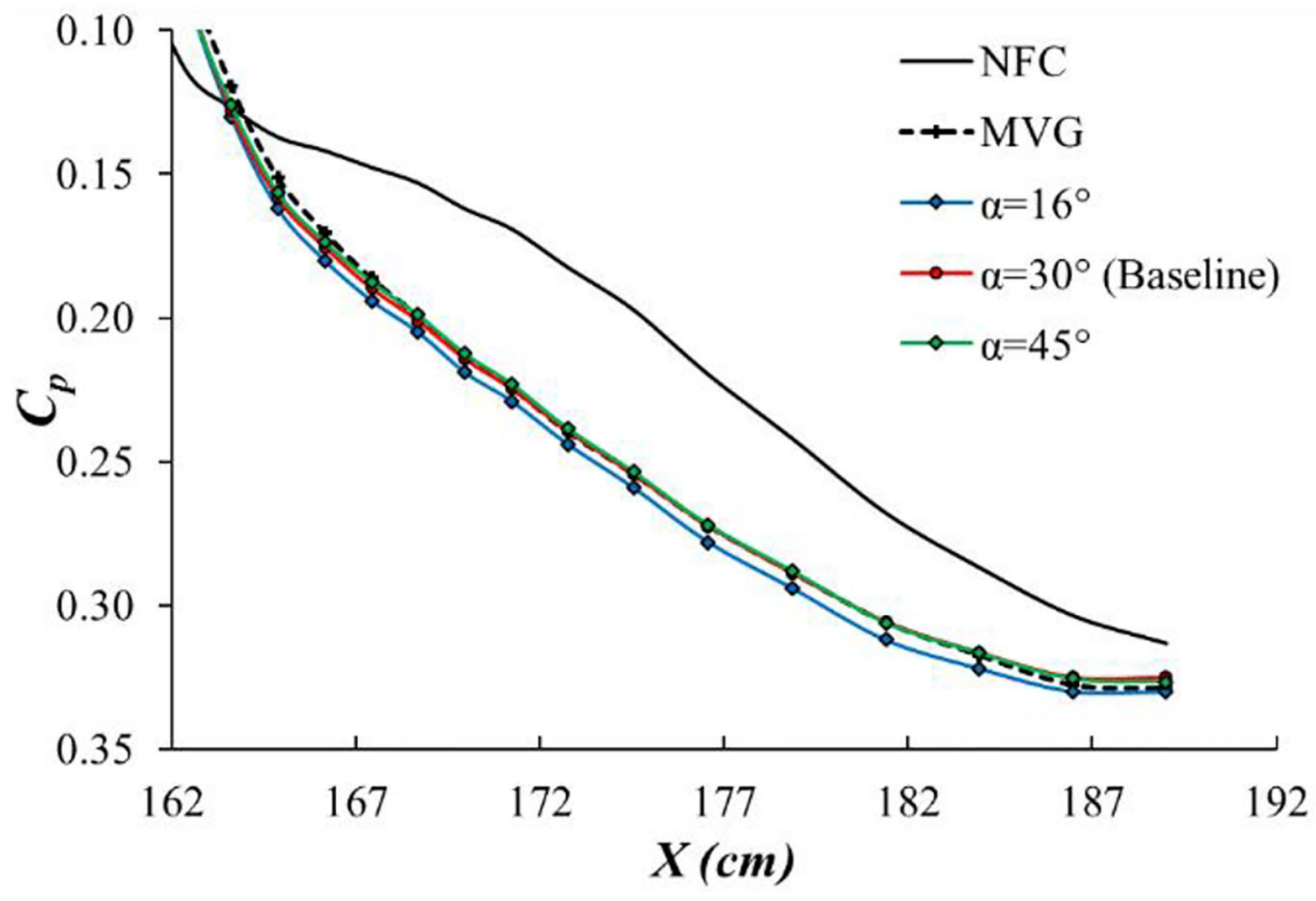

Fig. 16.

The effect of pitch angle ( $a$ ) on the $C_{p}$ distribution. 


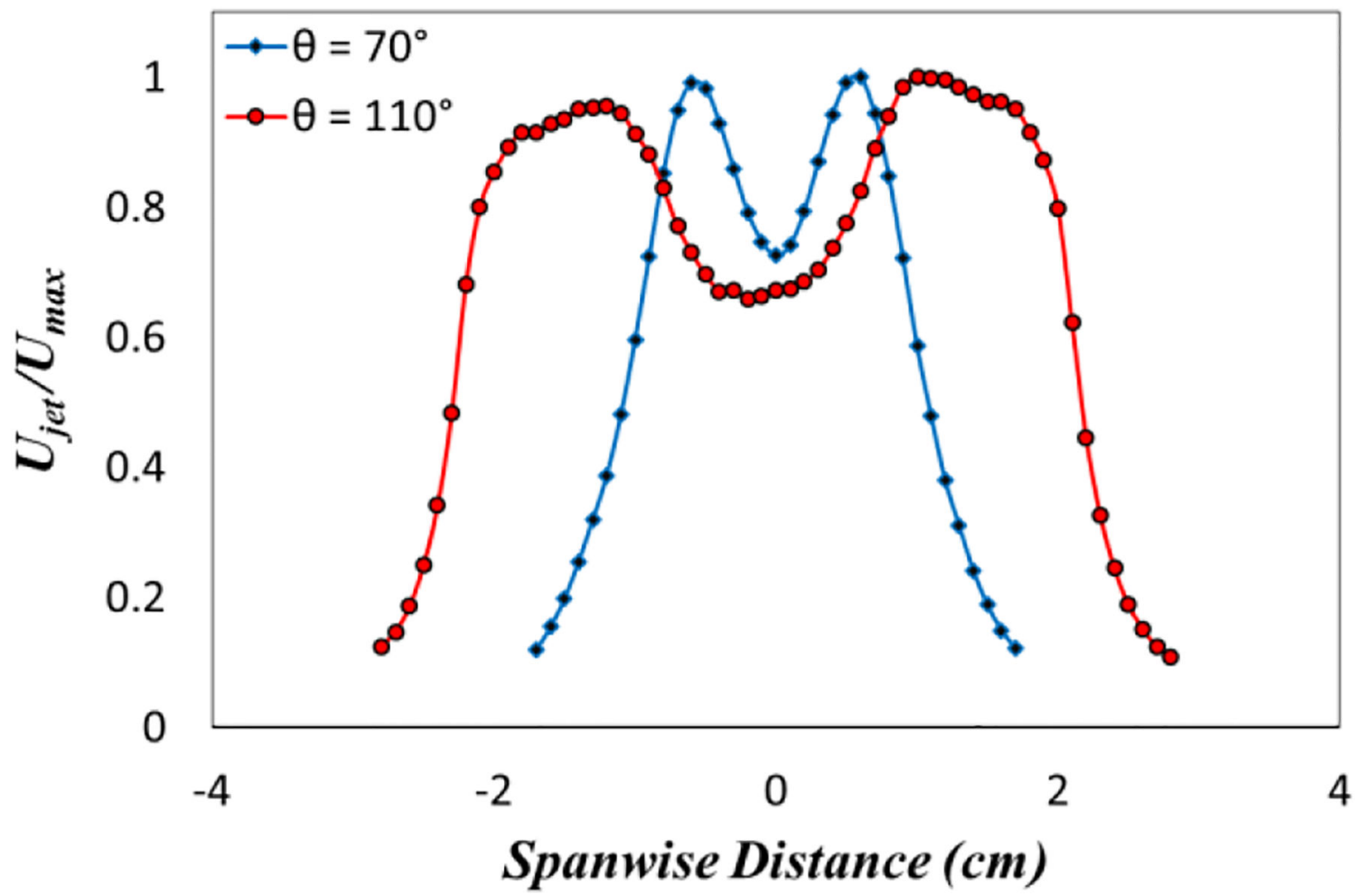

Fig. 17.

Time averaged jet velocity profiles for two actuators with different spreading angles. 


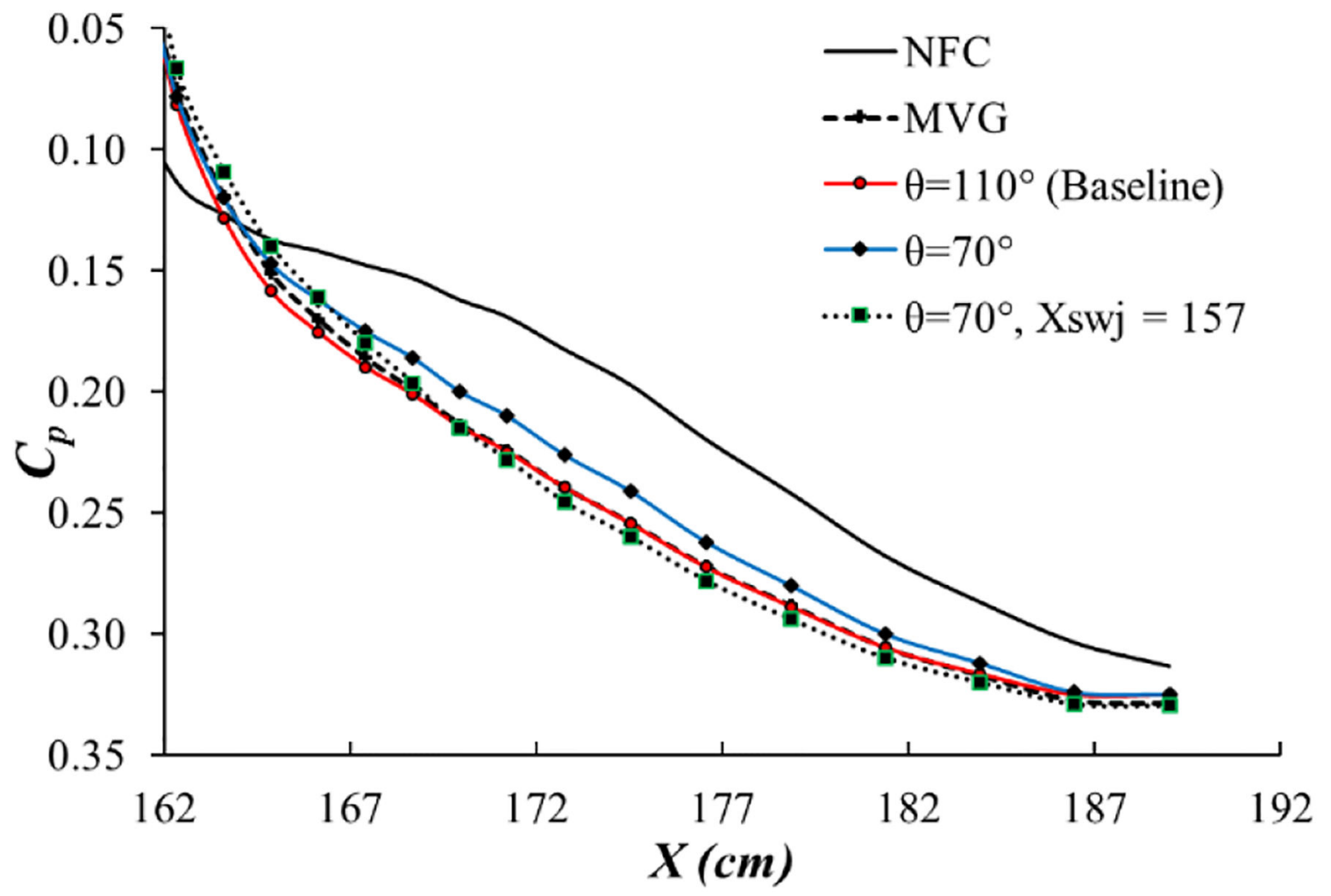

Fig. 18.

The effect of spreading angle $(\theta)$ on the $C_{p}$ distribution. 






Fig. 19.

Oilflow visualization for $\theta=70^{\circ}, X S W J=147$. 

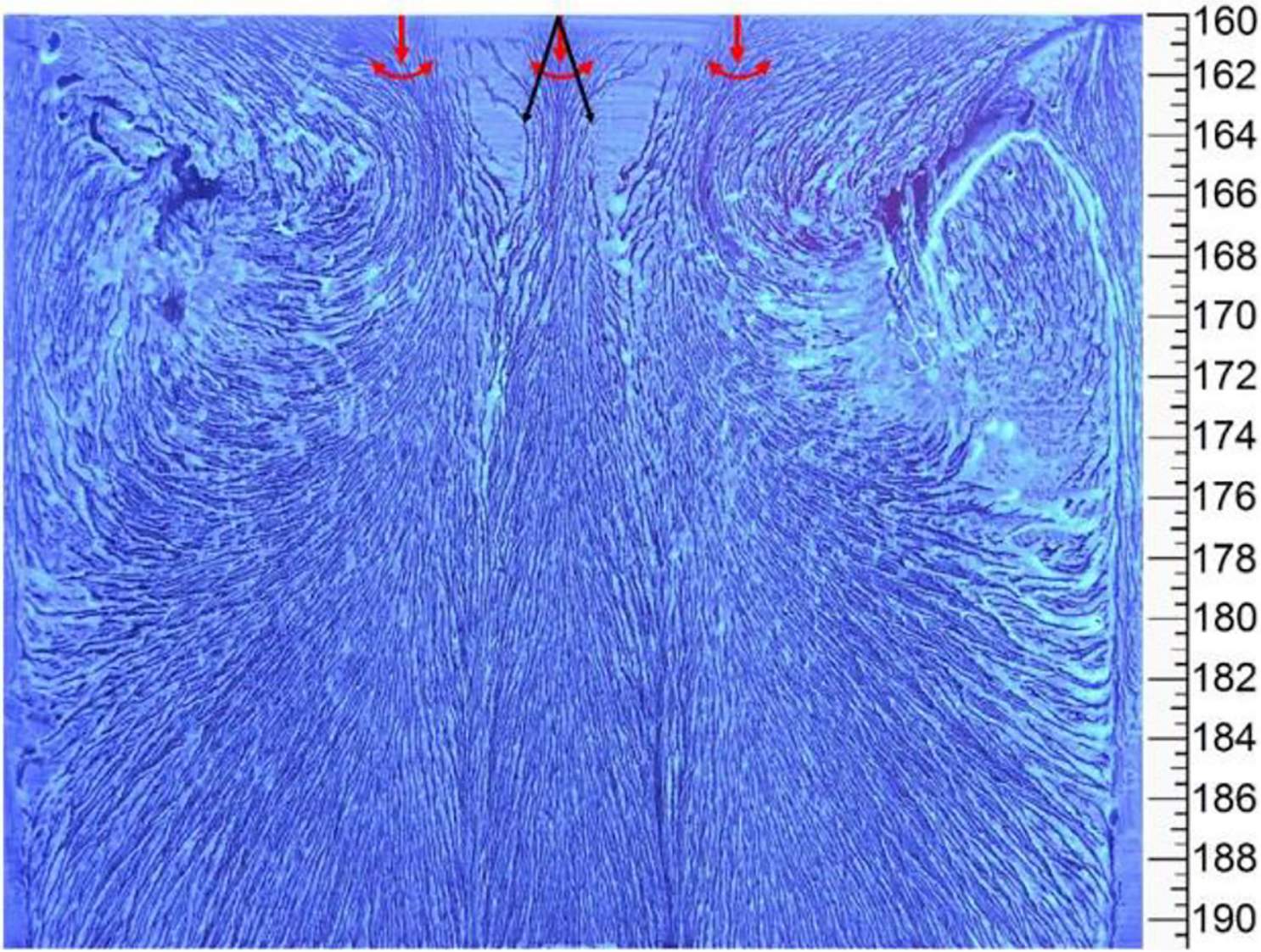

Fig. 20.

Oilflow visualization for $\theta=70^{\circ}, X S W J=157$.

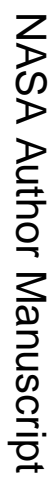




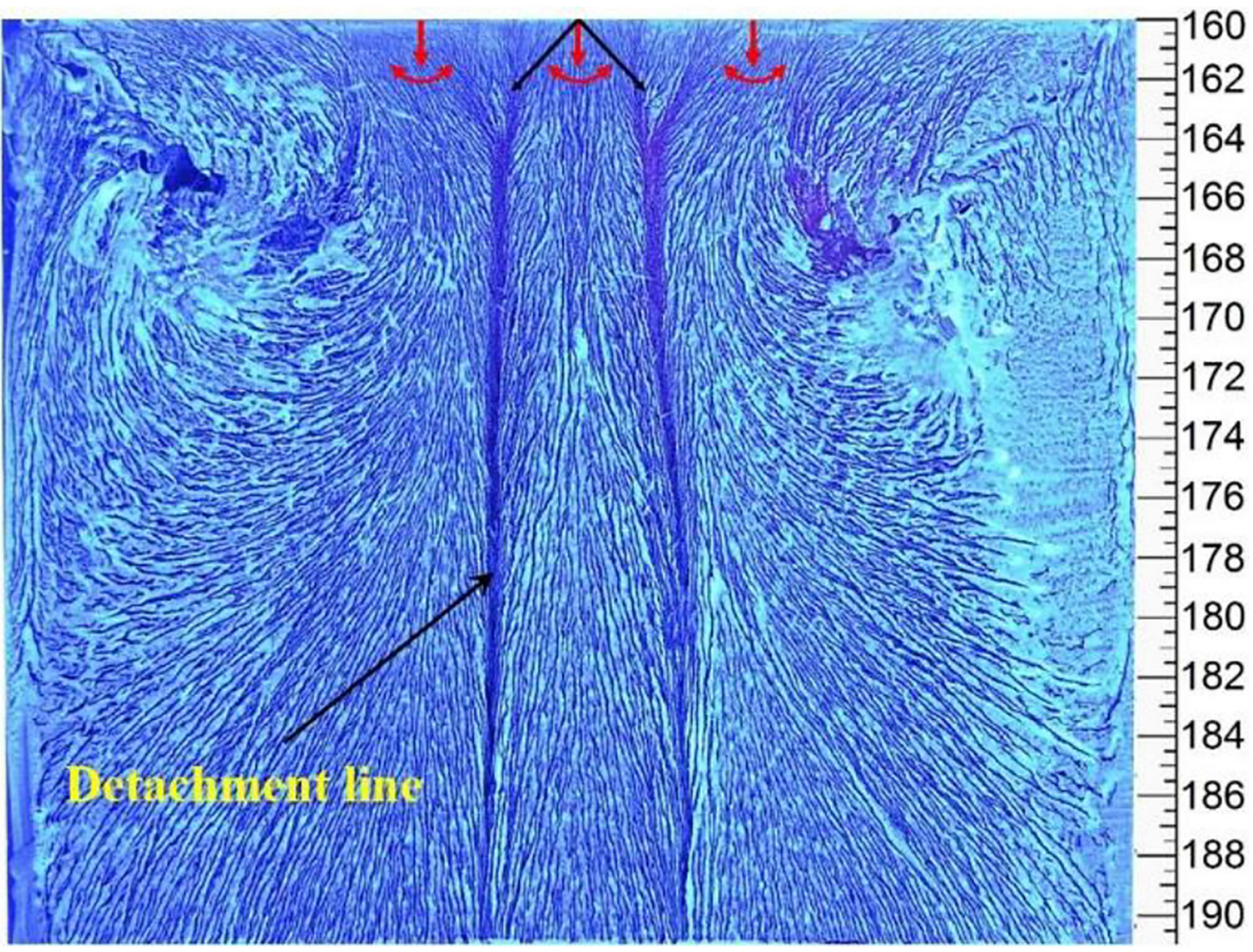

Fig. 21.

Oilflow visualization for $\theta=110^{\circ}, X S W J=157$ and $C_{\mu}=0.2 \%$. 


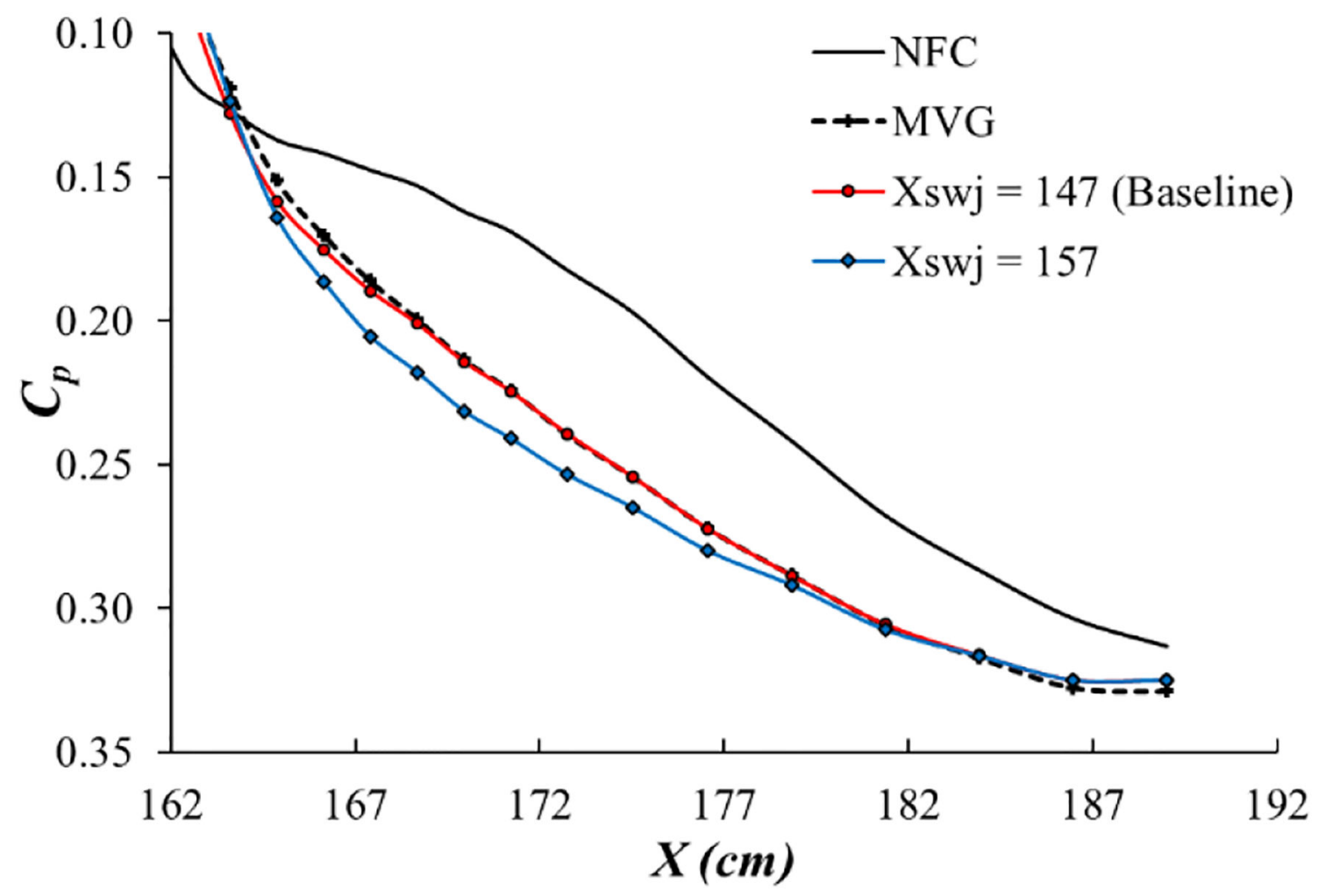

Fig. 22.

The effect of actuator location ( $X S W J$ ) on the $C_{p}$ distribution. 


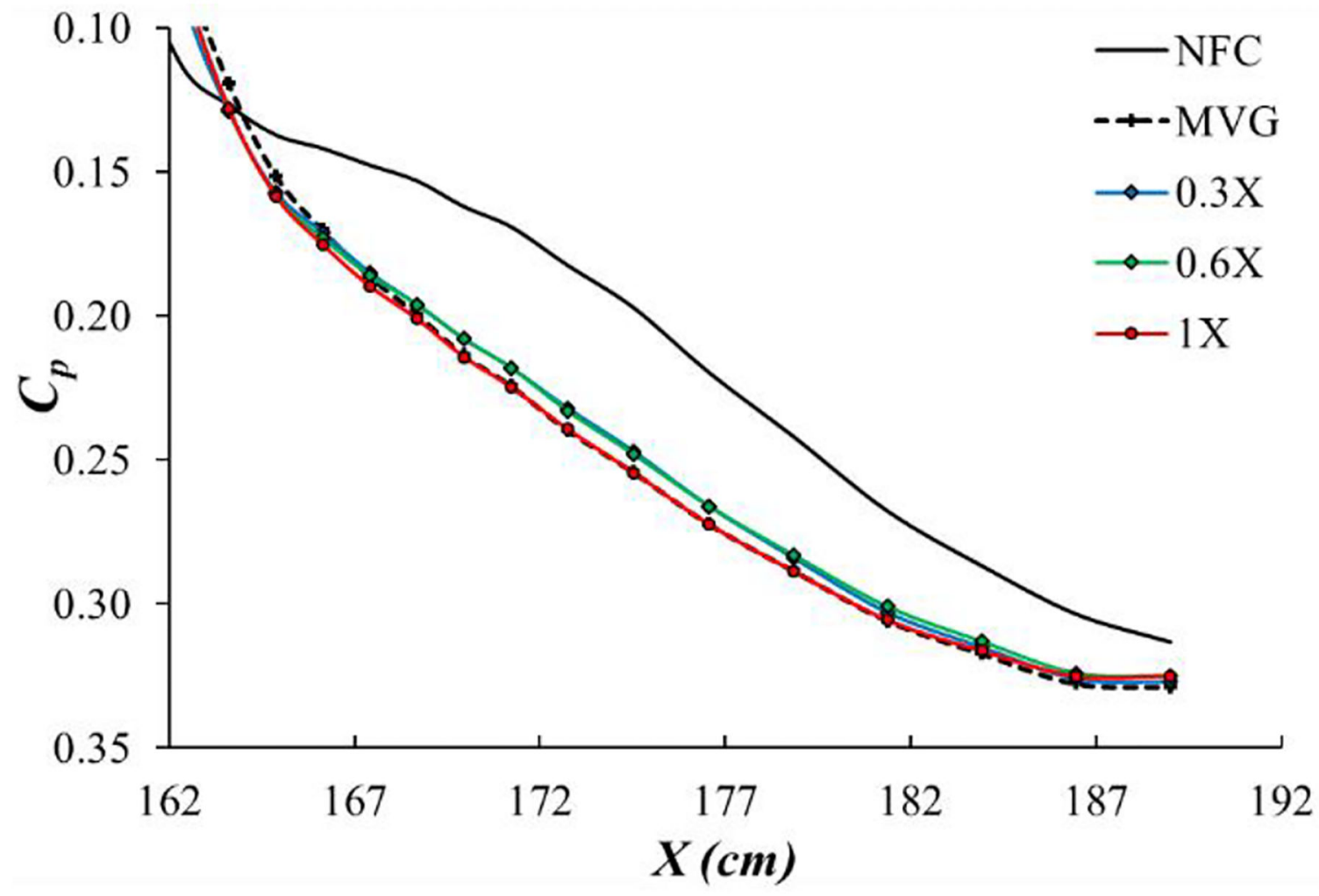

Fig. 23.

The effect of actuator size on the $C_{p}$ distribution for $C_{\mu}=0.4 \%$. 




Fig. 24.

The effect of actuator size on the $C_{p}$ distribution for $C_{\pi}=0.8 \%$. 


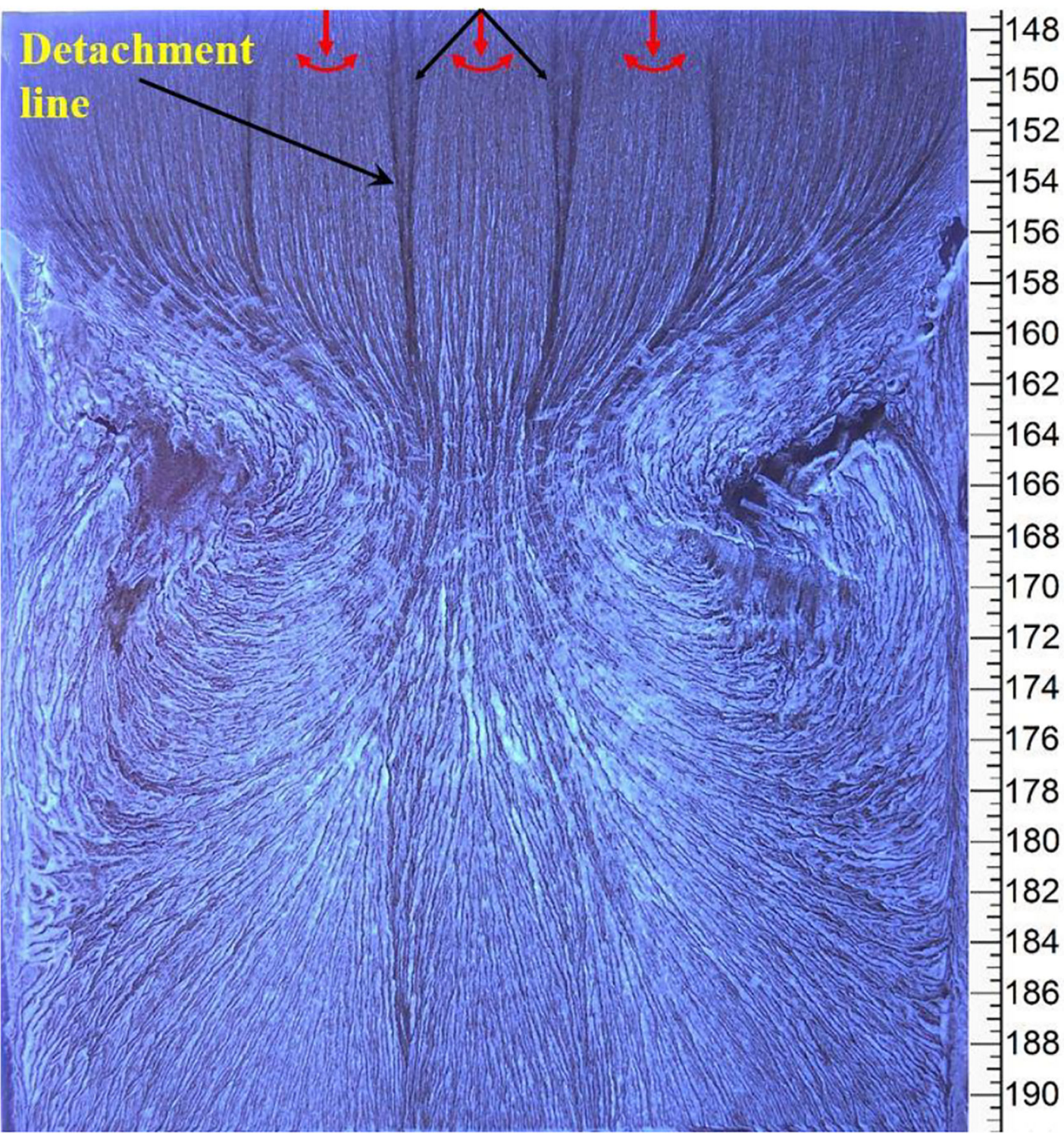

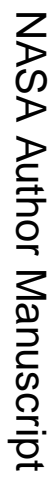

Fig. 25.

Oilflow visualization for $0.6 \mathrm{X}$ actuators. 


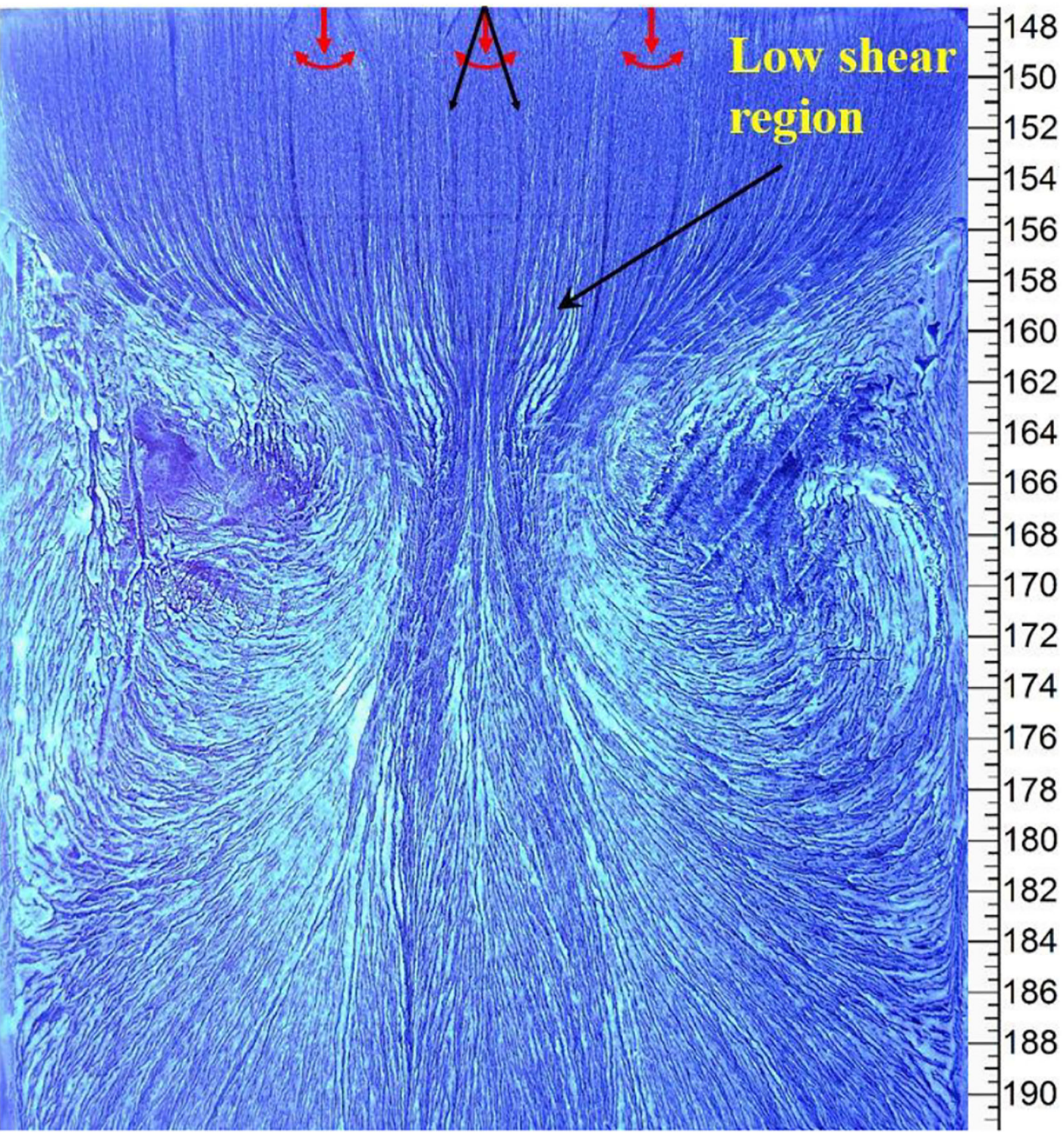

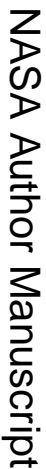

Fig. 26.

Oilflow visualization for $0.3 \mathrm{X}$ actuators. 




Fig. 27.

Time averaged jet velocity profiles for different sized actuators. 
Table 1.



Actuator parameters for different operation modes.

\begin{tabular}{ccccc}
\hline $\boldsymbol{C}_{\boldsymbol{\pi}}=\mathbf{0 . 8 \%}$ & $\boldsymbol{Q}(\boldsymbol{L} / \mathbf{s})$ & $\boldsymbol{V R}$ & $\boldsymbol{P}_{\boldsymbol{a c t}}(\boldsymbol{k P a})$ & $\boldsymbol{C}_{\boldsymbol{\pi}}(\boldsymbol{\%})$ \\
\hline SWJ & 4.15 & 1.6 & 6.96 & $0.40 \%$ \\
VGJ & 4.39 & 1.7 & 6.62 & $0.45 \%$ \\
STJ & 4.67 & 1.8 & 6.2 & $0.50 \%$ \\
\hline
\end{tabular}

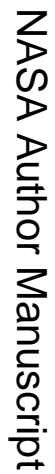

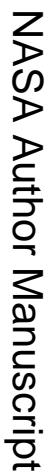

AIAA J. Author manuscript; available in PMC 2019 August 08. 
Table 2.



Variation of actuator parameters with scale for a fixed $C_{\mu}$.

\begin{tabular}{cccccc}
\hline $\mathbf{C}_{\boldsymbol{\pi}}=\mathbf{0 . 4 \%}$ & $\mathbf{Q}(\mathbf{L} / \mathbf{s})$ & $\mathbf{V R}$ & $\mathbf{P}_{\text {act }}(\mathbf{k P a})$ & $\mathbf{C}_{\boldsymbol{\pi}}(\%)$ & $\mathbf{f}(\mathbf{H z})$ \\
\hline $0.3 \mathrm{X}$ & 1.25 & 5.32 & 38.82 & 1.33 & 1537 \\
$0.6 \mathrm{X}$ & 2.48 & 2.65 & 15.93 & 1.09 & 406 \\
$1.0 \mathrm{X}$ & 4.14 & 1.59 & 6.9 & 0.78 & 178 \\
\hline
\end{tabular}

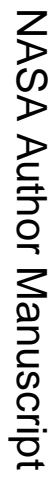

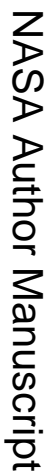

AIAA J. Author manuscript; available in PMC 2019 August 08. 
Table 3.

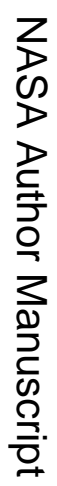

Variation of actuator parameters with scale for a fixed $C_{\pi}$.

\begin{tabular}{cccccc}
\hline $\mathbf{C}_{\boldsymbol{\pi}}=\mathbf{0 . 8 \%}$ & $\mathbf{Q}(\mathbf{L} / \mathbf{s})$ & $\mathbf{V R}$ & $\mathbf{P}_{\text {act }}(\mathbf{k P a})$ & $\mathbf{C}_{\boldsymbol{\mu}}(\%)$ & $\mathbf{f}(\mathbf{H z})$ \\
\hline $0.3 \mathrm{X}$ & 1.03 & 4.41 & 28.2 & 0.27 & 1325 \\
$0.6 \mathrm{X}$ & 2.27 & 2.42 & 12.76 & 0.33 & 375 \\
$1.0 \mathrm{X}$ & 4.17 & 1.61 & 6.96 & 0.40 & 178 \\
\hline
\end{tabular}

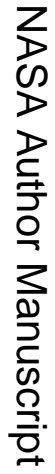

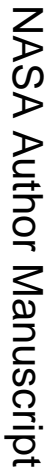

AIAA J. Author manuscript; available in PMC 2019 August 08. 